\title{
Analytical Model for the Impulse of Single-Cycle Pulse Detonation Tube
}

\author{
E. Wintenberger,* J. M. Austin, ${ }^{*}$ M. Cooper, ${ }^{\dagger}$ S. Jackson, ${ }^{*}$ and J. E. Shepherd \\ California Institute of Technology, Pasadena, California 91125
}

\begin{abstract}
An analytical model for the impulse of a single-cycle pulse detonation tube has been developed and validated against experimental data. The model is based on the pressure history at the thrust surface of the detonation tube. The pressure history is modeled by a constant pressure portion, followed by a decay due to gas expansion out of the tube. The duration and amplitude of the constant pressure portion is determined by analyzing the gasdynamics of the self-similar flow behind a steadily moving detonation wave within the tube. The gas expansion process is modeled using dimensional analysis and empirical observations. The model predictions are validated against direct experimental measurements in terms of impulse per unit volume, specific impulse, and thrust. Comparisons are given with estimates of the specific impulse based on numerical simulations. Impulse per unit volume and specific impulse calculations are carried out for a wide range of fuel-oxygen-nitrogen mixtures (including aviation fuels) of varying initial pressure, equivalence ratio, and nitrogen dilution. The effect of the initial temperature is also investigated. The trends observed are explained using a simple scaling analysis showing the dependency of the impulse on initial conditions and energy release in the mixture.
\end{abstract}

\begin{tabular}{lll} 
& \multicolumn{1}{c}{ Nomenclature } \\
$A$ & $=$ & cross-sectionalarea of detonation tube \\
$C^{ \pm}$ & $=$ & characteristics, left- and right-facing families \\
$\hat{C}^{-}$ & $=$ & first reflected characteristic to reach the thrust surface \\
$c_{1}$ & $=$ & sound speed of reactants \\
$c_{2}$ & $=$ & sound speed of burned gases just behind \\
& & detonation wave \\
$c_{3}$ & $=$ & sound speed of burned gases behind Taylor wave \\
$d$ & $=$ & inner diameter of detonation tube \\
$f$ & $=$ & cycle repetition frequency \\
$g$ & $=$ & standard Earth gravitational acceleration \\
$\mathcal{H}$ & $=$ & nondimensional heat release \\
$I$ & $=$ & single-cycle impulse \\
$I_{\mathrm{sp}}$ & $=$ & mixture-based specific impulse \\
$I_{\mathrm{spf}}$ & $=$ & fuel-based specific impulse \\
$I_{V}$ & $=$ & impulse per unit volume \\
$J^{-}$ & $=$ & Riemann invariant on a left-facing characteristic \\
$K$ & $=$ & proportionality coefficient \\
$L$ & $=$ & length of detonation tube \\
$\mathcal{L}$ & $=$ & critical length scale for deflagration-to-detonation \\
& $=$ & transition \\
$M$ & $=$ & total mass of initial combustible mixture within \\
& & detonation tube \\
$M_{\mathrm{CJ}}$ & $=$ & Chapman-Jouguet Mach number \\
$M_{f}$ & $=$ & initial mass of fuel within detonation tube \\
$P$ & $=$ & pressure \\
$P_{e}=$ & exhaust pressure \\
$P_{0}=$ & pressure outside detonation tube \\
$P_{1}=$ & initial pressure of reactants \\
$P_{2}$ & $=$ Chapman-Jouguet pressure \\
$P_{3}$ & $=$ & pressure of burned gases behind Taylor wave \\
& &
\end{tabular}

Received 10 September 2001; revision received 8 August 2002; accepted for publication 21 August 2002. Copyright (C) 2002 by California Institute of Technology. Published by the American Institute of Aeronautics and Astronautics, Inc., with permission. Copies of this paper may be made for personal or internal use, on condition that the copier pay the $\$ 10.00$ per-copy fee to the Copyright Clearance Center, Inc., 222 Rosewood Drive, Danvers, MA 01923; include the code 0748-465803 $\$ 10.00$ in correspondence with the CCC.

* Graduate Student, Aeronautics, Graduate Aeronautical Laboratories.

†raduate Student, Mechanical Engineering, Graduate Aeronautical Laboratories.

₹Professor, Aeronautics, Graduate Aeronautical Laboratories. Member AIAA.

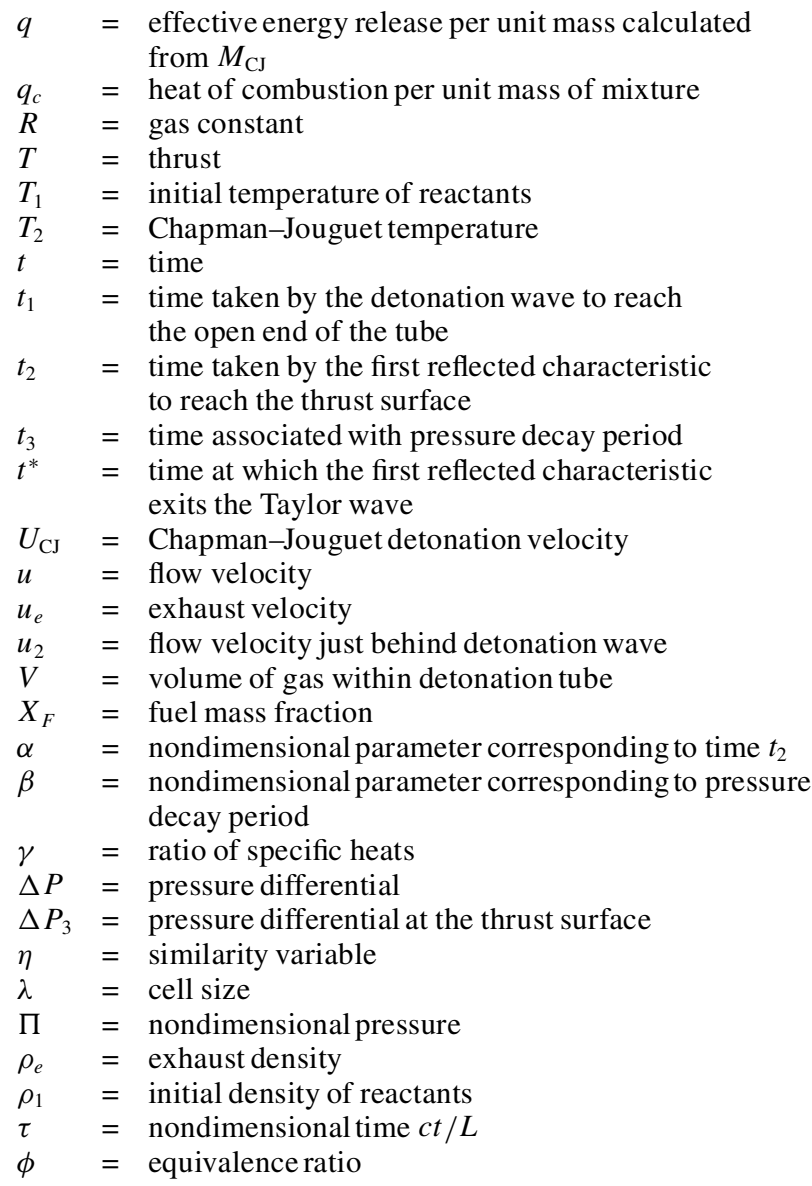

\section{Introduction}

KEY issue $\mathrm{e}^{1-5}$ in evaluating pulse detonation engine (PDE) propulsion concepts is reliable estimates of the performance as a function of operating conditions and fuel types. A basic PDE consists of an inlet, a series of valves, a detonation tube (closed at one end and open at the other), and an exit nozzle. It is an unsteady device that uses a repetitive cycle to generate thrust. The engine goes through four major steps during one cycle: the filling of the device with a combustible mixture, the initiation of the detonation near the 


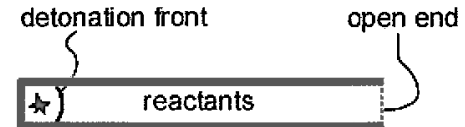

a)

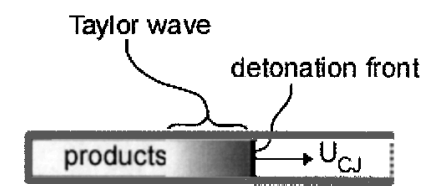

b)

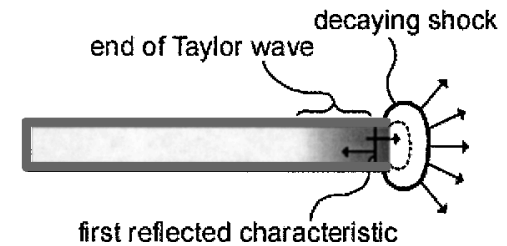

c)

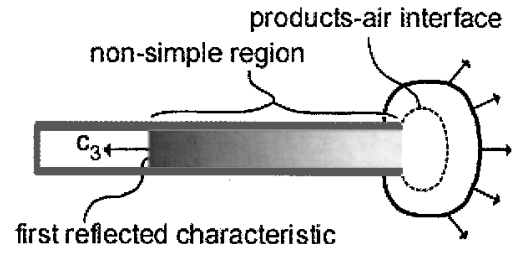

d)

Fig. 1 Pulse detonation engine cycle: a) detonation is initiated at the thrust surface; b) detonation, followed by the Taylor wave, propagates to the open end of the tube at a velocity $U_{\mathrm{CJ}}$; c) expansion wave is reflected at the mixture-air interface and immediately interacts with the Taylor wave while the products start to exhaust from the tube; and d) first characteristic of the reflected expansion reaches the thrust surface and decreases the pressure at the thrust surface.

closed end (thrust surface), the propagation of the detonation down the tube, and, finally, the exhaust of the productsinto the atmosphere. (Initiation at the closed end of the tube is not an essential part of PDE operation but greatly simplifies the analysis and will be used throughout the present study. Zhdan et al. ${ }^{6}$ found that the impulse is essentially independent of the igniter location for prompt initiation.) A schematic of the cycle for the detonation tube alone is shown in Fig. 1. The pressure differential created by the detonation wave on the tube's thrust surface produces unsteady thrust. If the cycle is repeated at a constant frequency, typically from 10 to $100 \mathrm{~Hz}$, an average thrust useful for propulsion is generated.

The goal of the present study is to provide a simple predictive model for detonation tube thrust. To do that, we have to carry out a fully unsteady treatment of the flow processes within the tube. This is a very different situation from modeling conventional propulsion systems such as turbojets, ramjets, and rockets for which steadystate, steady-flow analyses define performance standards. In those conventional systems, thermodynamic cycle analyses are used to derive simple but realistic upper bounds for thrust, thrust-specific fuel consumption, and other performance figures of merit. Because of the intrinsically unsteady nature of the PDE, the analogous thermodynamic bounds on performance have been elusive.

Unlike some previous ${ }^{2}$ and contemporary ${ }^{7}$ analyses, we do not attempt to replace the unsteady PDE cycle with a fictitious steadystate, steady-flow cycle. Although these analyses are purported to provide an ideal or upper bound for performance, we find that these bounds are so broad that they are unsuitable for making realistic performance estimates for simple devices like a detonation tube. This becomes clear when comparing the predicted upper bound values ${ }^{2,7}$ of 3000-5000 s for the fuel-based specific impulse of typical stoichiometric hydrocarbon-air mixtures with the measured values of about $2000 \mathrm{~s}$ obtained in detonation tube experiments. ${ }^{6-8-10}$ Instead, the present model focuses on the gasdynamic processes in the detonation tube during one cycle. The model is based on a physical description of the flow inside the tube and uses elementary onedimensional gasdynamics and dimensional analysis of experimen- tal observations. The model computes the impulse delivered during one cycle of operation as the integral of the thrust during one cycle.

It is critical to gain understanding of the single-cycle impulse of a detonation tube before more complex engine configurations are considered. There have been a number of efforts to develop a gasdynamics-basedmodel for single-cycle operation of detonation tubes. The pioneering work on single-cycle impulse was reported in 1957 by Nicholls et al., ${ }^{11}$ who proposed a very simplified model for the impulse delivered during one cycle. Only the contribution of the constant pressure portion at the thrust surface was considered, and the contribution of the pressure decay period was neglected. Consequently, their model predictions are about $20 \%$ lower than the results of our model presented here and the values obtained from modern experiments.

Zitoun and Desbordes ${ }^{8}$ proposed a model for the single-cycle impulse and compared this to their experimentally measured data. They showed predictions for stoichiometric mixtures of ethylene, hydrogen, and acetylene with oxygen and air. The models of Nicholls et al., ${ }^{11}$ Zitoun and Desbordes, ${ }^{8}$ and the more recent work of Endo and Fujiwara ${ }^{12}$ have many features in common with the present model because they are all based on a simple gasdynamic description of the flowfield. Zhdan et al. ${ }^{6}$ used both numerical simulations and simple analytical models, based on the results of Stanyukovich, ${ }^{13}$ to predict the impulse for tubes completely and partially filled with a combustible mixture.

In addition to analytical models, numerous numerical simulations have investigated various aspects of PDEs. Early studies, reviewed by Kailasanath et al., ${ }^{14}$ gave disparate and often contradictory values for performance parameters. Kailasanath and Patnaik ${ }^{5}$ identified how the issue of outflow boundary conditions can account for some of these discrepancies. With the recognition of this issue and the availability of high-quality experimental data, there is now substantial agreement ${ }^{15}$ between careful numerical simulation and experimental data, at least in the case of ethylene-air mixtures. However, even with improvements in numerical capability, it is desirable to develop simple analytical methods that can be used to rapidly and reliably estimate the impulse delivered by a detonation tube during one cycle to predict trends and to better understand the influence of fuel type, initial conditions, and tube size without conducting a large number of numerical simulations.

An end-to-end performance analysis of a PDE has to take into account the behavior of the inlet, the valves, the combustor, and the exit nozzle. However, the ideal performance is mainly dictated by the thrust generation in the detonation tube. In developing our model, we have considered the simplest configuration of a singlecycle detonation tube open at one end and closed at the other. We realize that there are significant issues ${ }^{3}$ associated with inlets, valves, exit nozzles, and multicycle operation that are not addressed in our approach. However, we are anticipating that our simple model can be incorporated into more elaborate models that will account for these features of actual engines and that the present model will provide a basis for realistic engine performance analysis.

The paper is organized as follows. First, we describe the flowfield for an ideal detonation propagating from the closed end of a tube toward the open end. We describe the essential features of the ideal detonation, the following expansion wave, and the relevant wave interactions. We present a simple numerical simulation illustrating these issues. Second, we formulate a method for approximating the impulse with a combination of analytical techniques and dimensional analysis. Third, the impulse model is validated by comparison with experimental data and numerical simulations. Fourth, a scaling analysis is performed to study the dependency of the impulse on initial conditions and energy release in the mixture. Fifth, the impulse model is used to compute impulse for a range of fuels and initial conditions. The influence of fuel type, equivalence ratio, initial pressure, and initial temperature are examined in a series of parametric computations.

\section{Flowfield Associated with an Ideal Detonation in a Tube}

The gasdynamic processes that occur during a single cycle of a PDE can be summarized as follows. A detonation wave is directly 
initiated and propagates from the thrust surface toward the open end. For the purposes of formulating our simple model, we consider ideal detonations described as discontinuities propagating at the Chapman-Jouguet (CJ) velocity. The detonation front is immediately followed by a self-similar expansion wave ${ }^{16,17}$ known as the Taylor wave. ${ }^{17}$ This expansion wave decreases the pressure and brings the flow to rest. The method of characteristics ${ }^{16,17}$ can be used to calculate flow properties within the Taylor wave. [See Eqs. (11-13) in the following section.]

There is a stagnant region extending from the rear of the Taylor wave $^{17}$ to the closed end of the tube. When the detonationreaches the open end of the tube, a shock is generated and diffracts out into the surrounding air. Because the pressure at the tube exit is higher than ambient, the transmitted shock continues to expand outside of the tube. Because the flow at the tube exit is subsonic, a reflected wave propagates back into the tube. This reflected wave is usually an expansion wave, which reflects from the closed end, reducing the pressure and creating an expansion wave that propagates back toward the open end. After several sequences of wave propagation within the tube, the pressure inside approaches atmospheric. A simplified, but realistic, model of the flowfield can be developed by using classical analytical methods.

\section{Ideal Detonation and Taylor Wave ${ }^{17}$}

To predict the ideal impulse performance of a pulsed detonation tube, we can consider the detonation as a discontinuity that propagates with a constant velocity. This velocity is a function of the mixture composition and initial thermodynamic state. The reaction zone structure and the associated property variations such as the von Neumann pressure spike are neglected in this model because the contribution of these features to the impulse is negligible.

The detonation speed is determined by the standard CJ model of a detonation that assumes that the flow just downstream of the detonation is moving at sonic velocity relative to the wave. This special downstream state, referred to as the CJ point, can be found by numerically solving the relations for mass, momentum, and energy conservationacross the discontinuity while simultaneouslydetermining the chemical composition. Equilibrium computations ${ }^{18}$ based on realistic thermochemical properties and a mixture of the relevant gas species in reactants and products are used to calculate the chemical composition.

Alternatively, the conservation equations can be analytically solved for simple models, using an ideal gas equation of state, a fixed heat of reaction, and heat capacities that are independent of temperature. A widely used version of this model, described by Thompson, ${ }^{19}$ uses different properties in the reactants and products and a fixed value of the energy release $q$ within the detonation wave. In the present study, we use an even simpler version, ${ }^{20}$ the one- $\gamma$ model, which neglects the differences in specific heat and molar mass between reactants and products.

\section{Interaction of the Detonation with the Open End}

The flow behind a CJ detonation wave is subsonic relative to the tube and has a Mach number $M_{2}=u_{2} / c_{2}$ of approximately 0.8 for typical hydrocarbon mixtures. Hence, when the detonation wave reaches the open end, a disturbance propagates back into the tube in the form of a reflected wave..$^{21}$ The interface at the open end of the tube can be modeled in one dimension as a contact surface. When the detonation wave is incident on this contact surface, a transmitted wave will propagate out of the tube, and a reflected wave propagates into the tube toward the thrust surface.

The reflected wave can be either a shock or an expansion wave. A simple way to determine the nature of the reflected wave is to use a pressure-velocity diagram ${ }^{21}$ because the pressure and velocity must be matched across the contact surface after the interaction. In the case of a detonation wave exiting into air, the transmitted wave will always be a shock wave; the locus of solutions (the shock adiabat) is shown in Figs. 2 and 3. The shock adiabat is computed from the shock jump conditions, which can be written in terms of the pressure jump and velocity jump across the wave

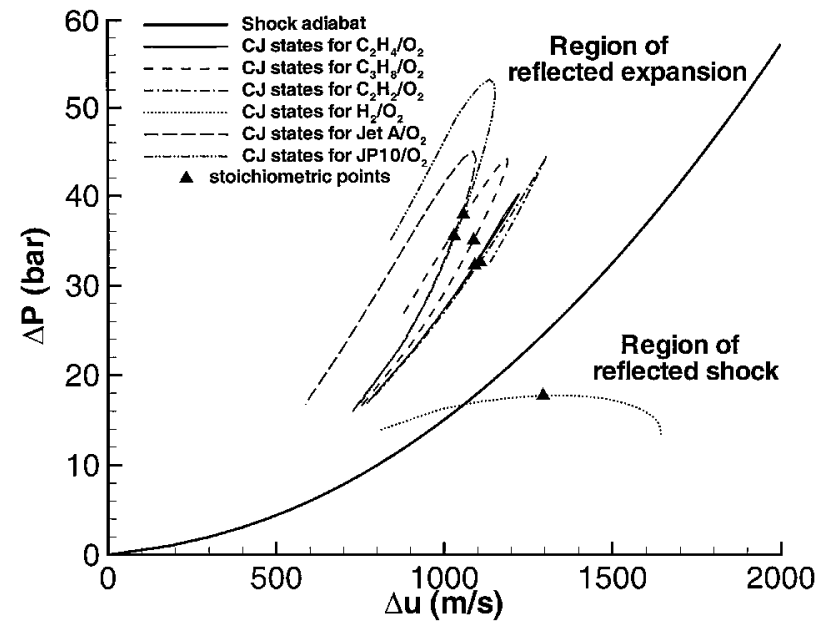

Fig. 2 Pressure-velocity diagram used to compute wave interactions at the tube open end for fuel-oxygen mixtures.

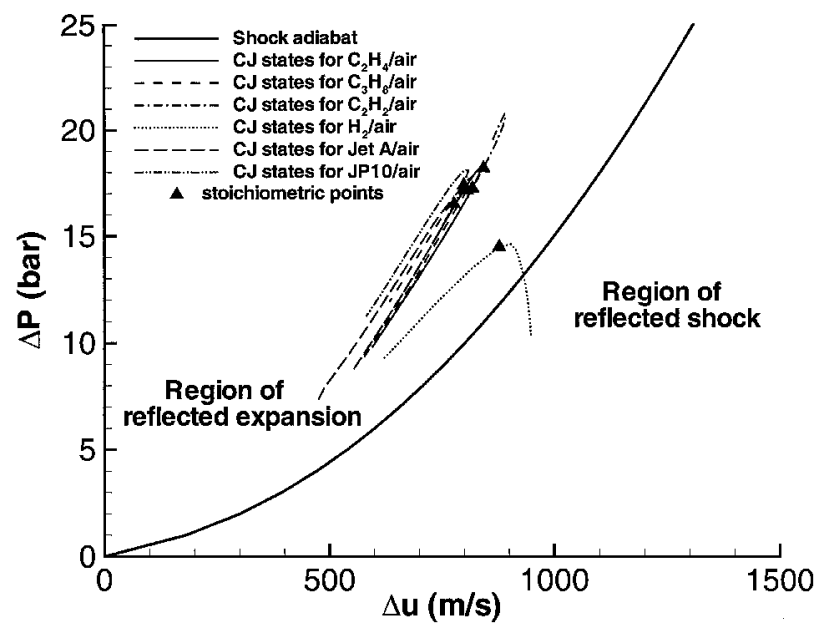

Fig. 3 Pressure-velocity diagram used to compute wave interactions at the tube open end for fuel-air mixtures.

$$
\frac{\Delta u}{c_{1}}=\frac{\Delta P}{P_{1}} / \gamma\left(1+\frac{\gamma+1}{2 \gamma} \frac{\Delta P}{P_{1}}\right)^{\frac{1}{2}}
$$

The reflected wave initially propagates back into the products at the CJ state behind the detonation wave. The CJ states for various fuels and equivalence ratios appear in Figs. 2 and 3. If the CJ point is below the shock adiabat, the reflected wave must be a shock to increase the pressure to match that behind the transmitted shock. Alternatively, if the CJ state is above the shock adiabat, the reflected wave must be an expansion to decrease the pressure to match that behind the transmitted shock. Hydrocarbon fuels all produce a reflected expansion wave at the tube's open end for any stoichiometry. However, a reflected shock is obtained for hydrogen-oxygen at an equivalence ratio $\phi>0.8$ (Fig. 2) and for very rich hydrogen-air mixtures with $\phi>2.2$ (Fig. 3).

Ultimately, following the initial interaction of the detonation wave with the contact surface, the pressure at the exit of the tube will drop as the transmitted shock wave propagates outward. In all cases, because the flow outside the tube is expanding radially behind the diffracting shock wave, an expansion wave also exists in the flow external to the tube. The flow in this region can not be modeled as one-dimensional. A numerical simulation (discussed hereafter) is used to illustrate this portion of the flow.

\section{Waves and Space-Time Diagram}

A space-time $(x-t)$ diagram, shown in Fig. 4, is used to present the important features of the flow inside the tube. The $x-t$ diagram 
displays the detonation wave propagating at the CJ velocity $U_{\mathrm{CJ}}$ followed by the Taylor wave. ${ }^{17}$ The first characteristic $\hat{C}^{-}$of the wave reflected from the mixture-air interface at the open end of the tube is also shown. The initial slope of this characteristic is determined by the conditions at the mixture-air interface and is then modified by interaction with the Taylor wave. After passing through the Taylor wave, the characteristic $\hat{C}^{-}$propagates at the sound speed $c_{3}$. The region lying behind this first characteristic is nonsimple because of the interaction between the reflected expansion wave and the Taylor wave. Two characteristic times can be defined: $t_{1}$, corresponding to the interaction of the detonation wave with the open end, and $t_{2}$, corresponding to the time necessary for the characteristic $\hat{C}^{-}$to reach the thrust surface. The diffracted shock wave in Fig. 4 is shown outside the tube as a single trajectory; however, this is actually a three-dimensional wave front that can not be fully represented on this simple plot.

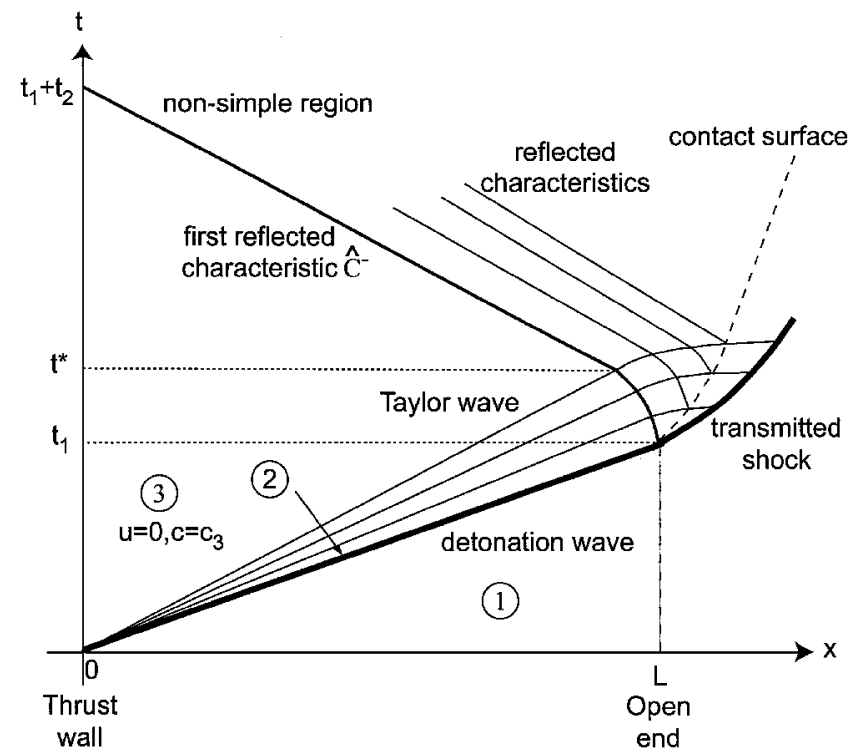

Fig. 4 Space-time diagram for detonation wave propagation and interaction with the tube's open end.

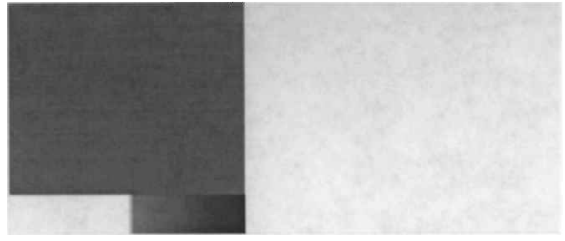

a) $t=t_{1}$

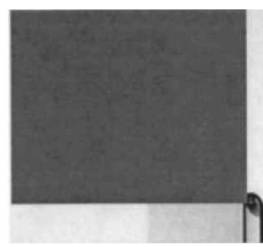

b) $t=1.11 t_{1}$

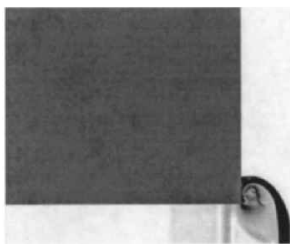

c) $t=1.32 t_{1}$

\section{Numerical Simulation Example}

To further examine the issues related to the interaction of the detonation with the open end of the tube, the flow was investigated numerically by $\mathrm{H}$. Hornung at the Graduate AeronauticalLaboratories, California Institute of Technology, Pasadena, California, in August 2000 using AMRITA. ${ }^{22}$ The Taylor wave similarity solution ${ }^{16,17}$ was used as an initial condition, assuming the detonation has just reached the open end of the tube when the simulation is started. This solution was calculated using a one- $\gamma$ model for detonations ${ }^{19,20}$ for a nondimensionalenergy release $q / R T_{1}=40$ across the detonation and $\gamma=1.2$ for reactants and products. The corresponding CJ parameters are $M_{\mathrm{CJ}}=5.6$ and $P_{\mathrm{CJ}} / P_{1}=17.5$, values representative of stoichiometric hydrocarbon-air mixtures.

The initial pressure $P_{1}$ ahead of the detonation wave was taken to be equal to the pressure $P_{0}$ outside the detonation tube. The simulation solved the nonreactive Euler equations using a KappaMUSCL-HLLE solver in the two-dimensional (cylindrical symmetry) computational domain, consisting of a tube of length $L$ closed at the left end and open to a half-space at the right end. Numerical schlieren images are displayed in Fig. 5, and the corresponding pressure and horizontal velocity profiles along the tube centerline are shown in Figs. 6 and 7, respectively. Only one-half of the tube is shown in Fig. 5; the lower boundary is the axis of symmetry of the cylindrical detonation tube. The times given in these Figs. 5-7 account the initial detonation travel from the closed end to the open end of the tube, so that the Figs. 5a, 6a, and 7a corresponds to a time $t_{1}=L / U_{\mathrm{CJ}}$.

Figures $5 \mathrm{a}, 6 \mathrm{a}$, and $7 \mathrm{a}$ show the initial condition with the pressure decreasing behind the detonation front from the CJ pressure $P_{2}$ to a value $P_{3}$ at the end of the Taylor wave. ${ }^{17}$ The detonation wave becomes a decaying shock as it exits the tube because the region external to the tube is nonreactive, simulating the surrounding atmosphere of most experimental configurations.

This decaying shock is initially planar, but is affected by the expansions originating from the corners of the tube and gradually becomes spherical. The pressure profiles show the decay of the pressure behind the leading shock front with time. A very complex flow structure, involving vortices and secondary shocks, forms behind the leading shock. The fluid just outside the tube accelerates due to the expansion waves coming from the corners of the tube. At the same time, the leading shock front exits the tube, a reflected expansion

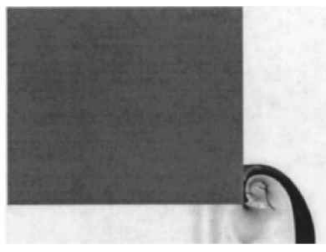

d) $t=1.47 t_{1}$

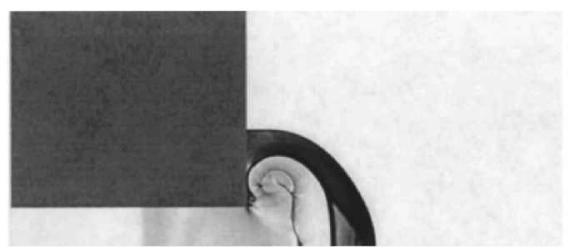

e) $t=1.95 t_{1}$

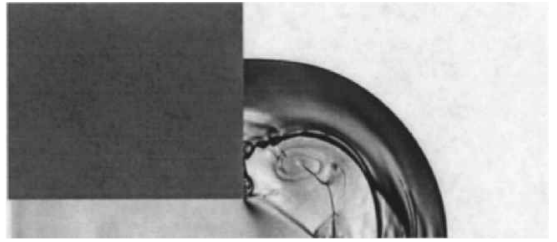

f) $t=2.81 t$

Fig. 5 Numerical schlieren images of the exhaust process. 


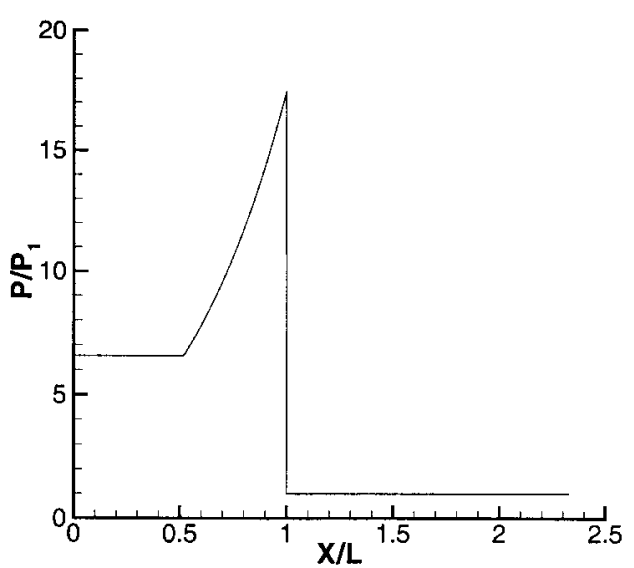

a) $t=t_{1}$

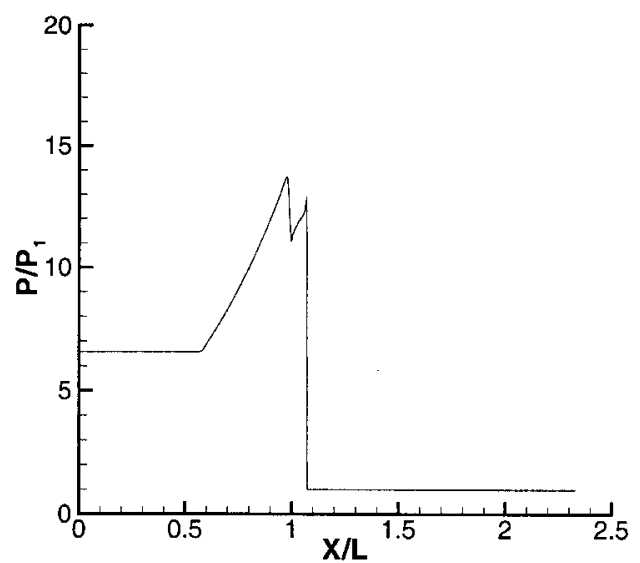

b) $t=1.11 t_{1}$

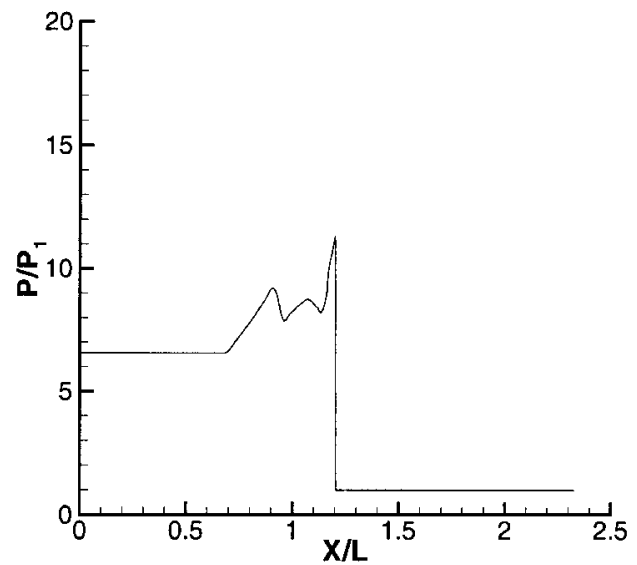

c) $t=1.32 t_{1}$

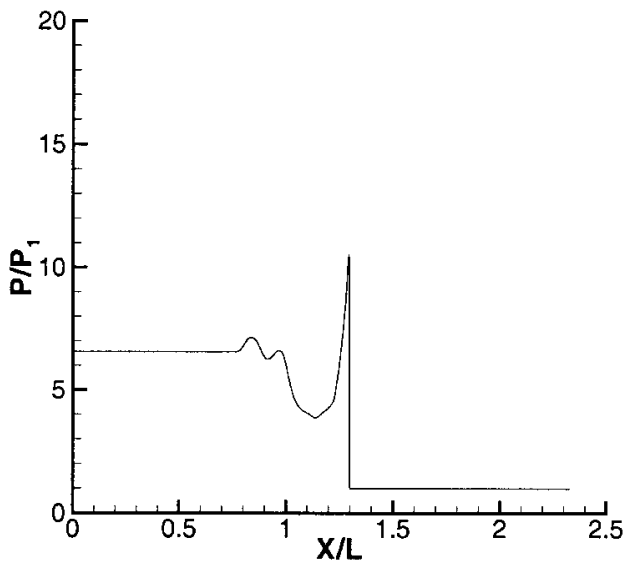

d) $t=1.47 t_{1}$

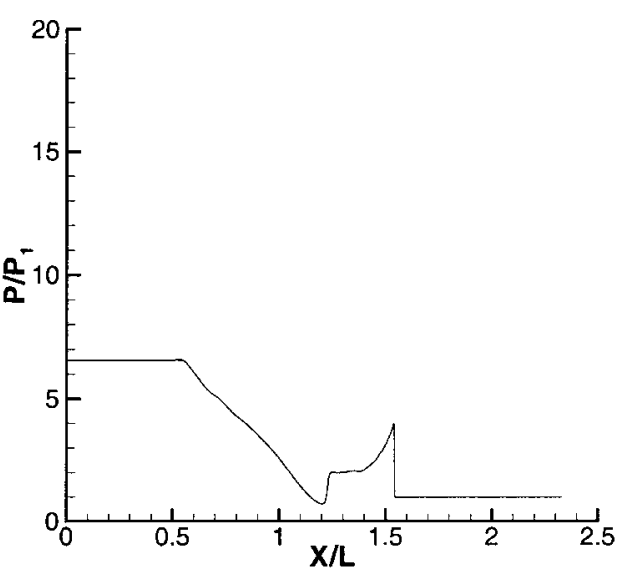

e) $t=1.95 t_{1}$

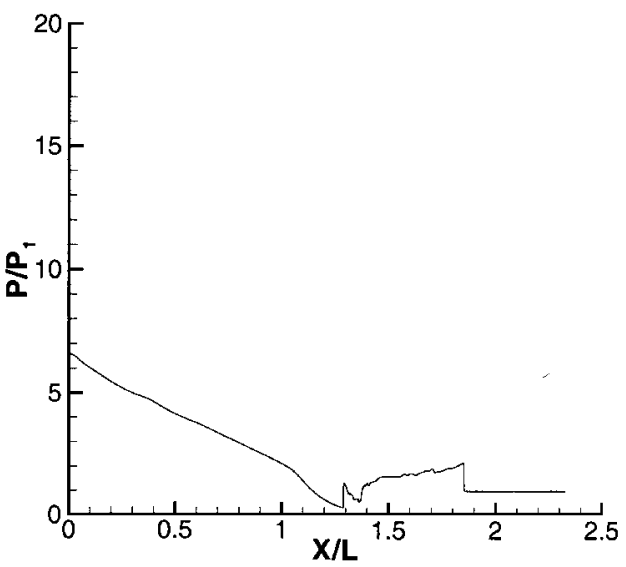

f) $t=2.81 t_{1}$

Fig. 6 Pressure along the tube centerline from numerical simulation; $P_{1}$ is the initial pressure inside and outside the tube.

wave is generated, and this wave propagates back into the tube, interacting with the Taylor wave. ${ }^{17}$ This reflected wave propagates until it reaches the closed end of the tube, decreasing the pressure and accelerating the fluid toward the open end. The exhaust process is characterized by low pressure and high flow velocity downstream of the tube exit. A system of quasi-steady shocks similar to those observed in steady underexpanded supersonic jets, and an unsteady leading shock wave, bring the flow back to atmospheric pressure.

One of the most important points learned from this simulation is that the flow inside the tube is one-dimensional, except for within one to two diameters of the open end. Another is that the pressure at the open end is unsteady, initially much higher than ambient pressure, and that it decreases at intermediate times to lower than ambient before finally reaching equilibrium. Despite the one-dimensional nature of the flow within the tube, it is important to simulate the multidimensional flow properly in the vicinity of the exit to get a realistic representation of the exhaust process. In our simple model, this is accomplished by using a nondimensional correlation of the experimental data for this portion of the process.

The normalized pressure $P / P_{1}$ at the thrust surface, as well as the normalized impulse per unit volume $(I / V)\left(U_{\mathrm{CJ}} / P_{1}\right)$ are shown as a function of normalized time $t / t_{1}$ in Fig. 8. The impulse per unit volume was computed by integrating the pressure at the thrust surface over time. Note that Figs. 5-8 take into account the initial detonation travel from the closed end to the open end of the tube. The pressure at the thrust surface remains constant until the reflected wave from the tube's open end reaches the thrust surface at time $t_{1}+t_{2} \approx 2.81 t_{1}$. The final pressure decay process is characterizedby 


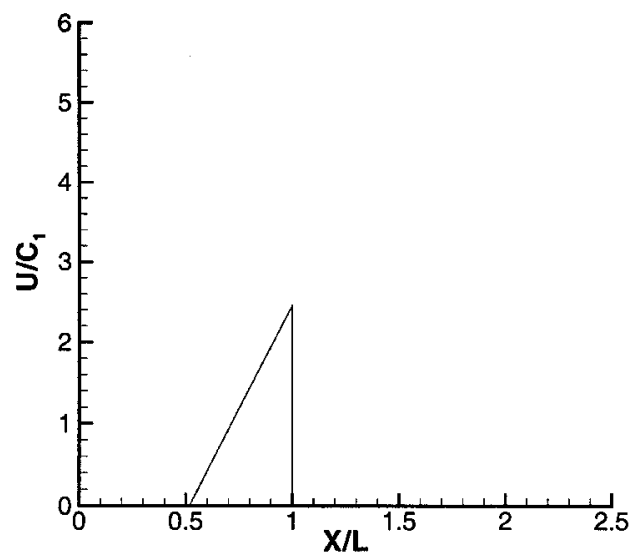

a) $t=t_{1}$

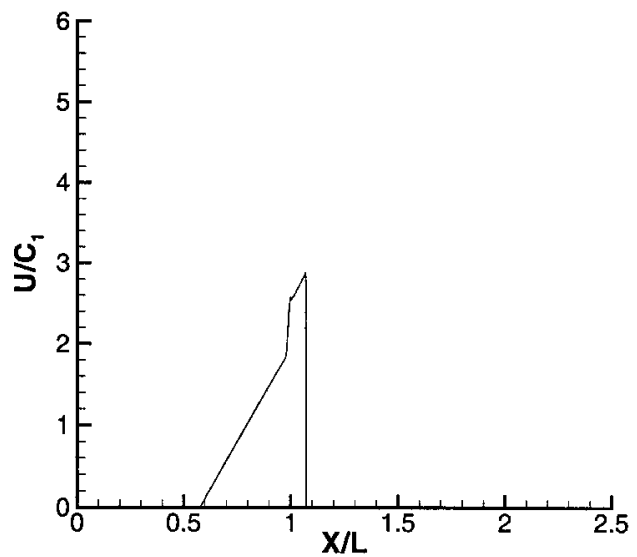

b) $t=1.11 t_{1}$

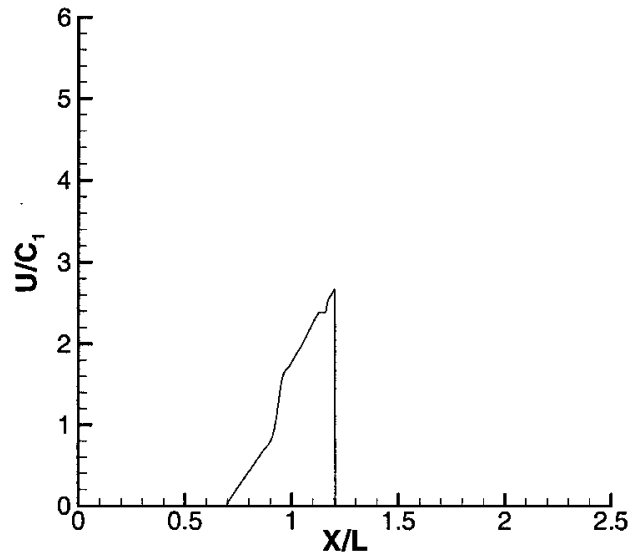

c) $t=1.32 t_{1}$

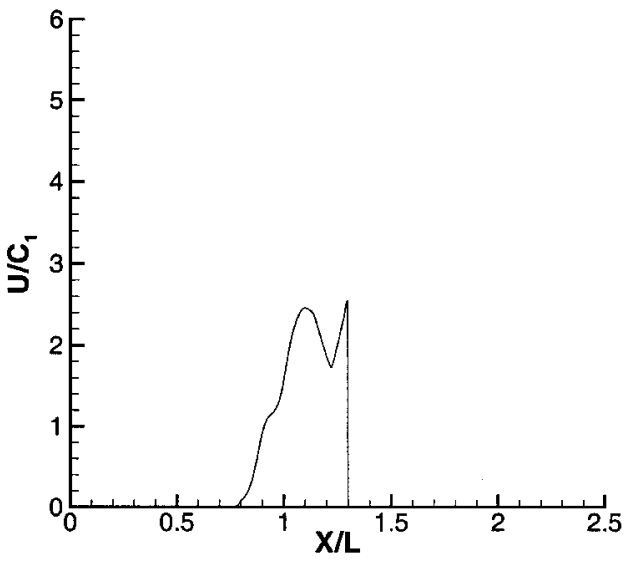

d) $t=1.47 t_{1}$

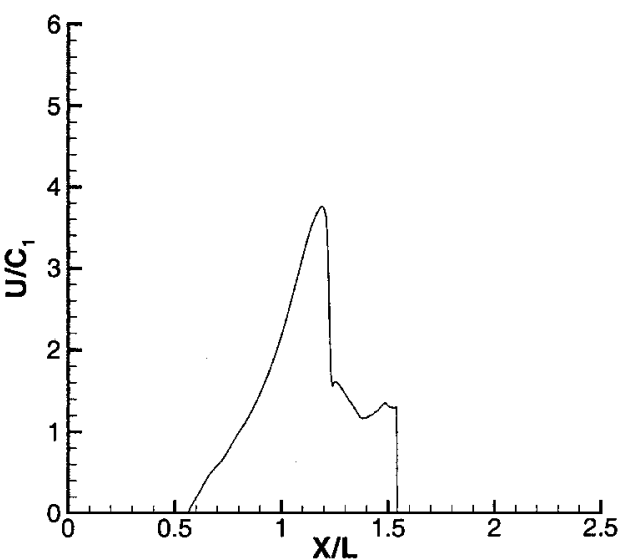

e) $t=1.95 t_{1}$

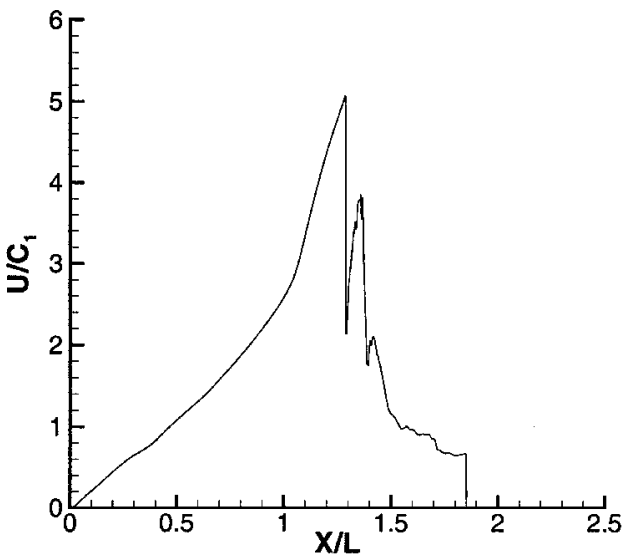

f) $t=2.81 t_{1}$

Fig. 7 Velocity along the tube centerline from numerical simulation; $c_{1}$ is the initial sound speed inside and outside the tube.

a steep pressure decrease and a region of subatmospheric pressure. The integratedimpulse consequentlyincreases to a maximumbefore decreasing due to this region of negative overpressure.

\section{Impulse Model}

Our impulse model is based on elementary gasdynamic considerations. We assume one-dimensional, adiabatic flow in a straight, unobstructed tube closed at one end and open at the other. The impulse is calculated by considering a control volume around the straight tube as shown in Fig. 9b. Figure 9a, which represents the usual control volume used for rocket engine analysis, requires the knowledge of the exit pressure $P_{e}$, the exhaust velocity $u_{e}$, and exhaust density $\rho_{e}$ (or mass flow rate). Figure $9 \mathrm{~b}$, the control volume considered in the model, requires only the knowledge of the pressure history at the thrust surface. The impulse is obtained by integrating the pressure differential $P_{3}-P_{0}$ across the thrust surface during one cycle, assuming $P_{e}=P_{0}$. This approach is rather limited and is certainly not applicable to airbreathing engines with complex inlets and/or exits. However, it is appropriate for a single tube of constant area, and the modeling assumptions eliminate the need for numerical simulations or detailed flow measurements required to evaluate the thrust by integration over the flow properties at the exit plane.

We have made a number of other simplifying assumptions. Nonideal effects such as viscosity or heat transfer are not considered. The detonation properties are calculated assuming the ideal onedimensional CJ profile. Real-gas thermodynamics are used to calculate the $\mathrm{CJ}$ detonation properties, and classical gasdynamics for a perfect gas are used to model the flow behind the detonation wave. 

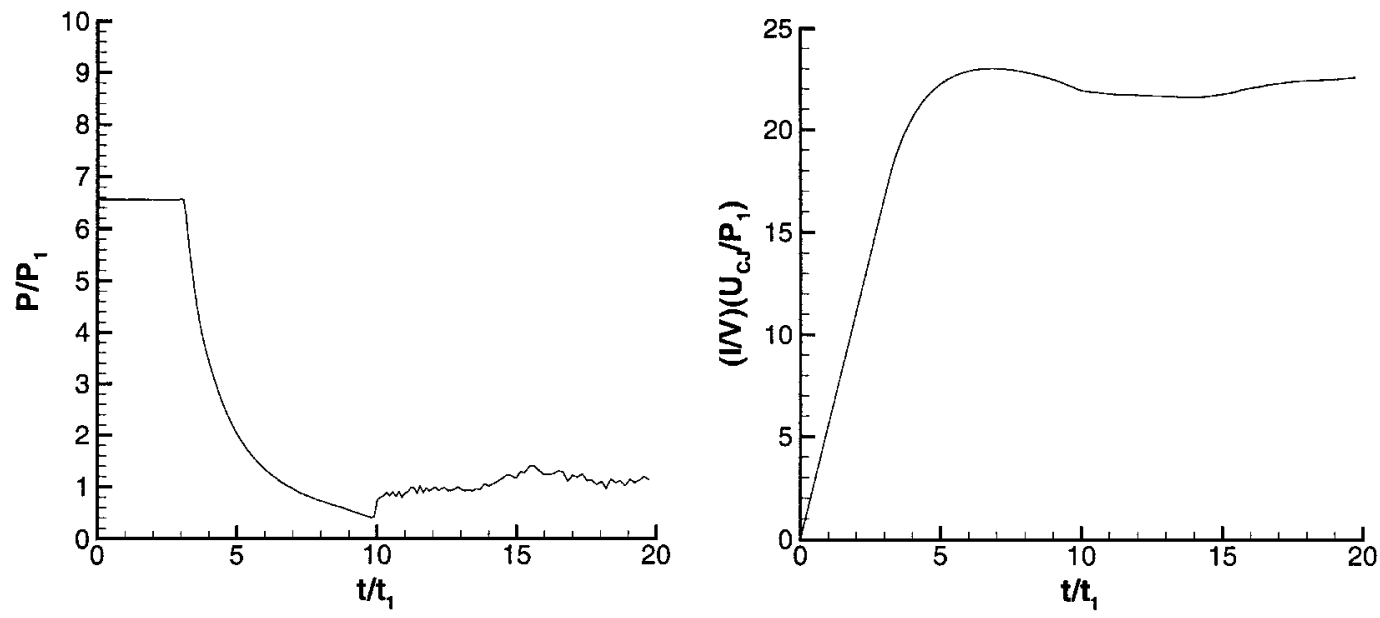

Fig. 8 Nondimensionalized thrust surface pressure and impulse per unit volume as a function of nondimensionalized time for the numerical simulation.

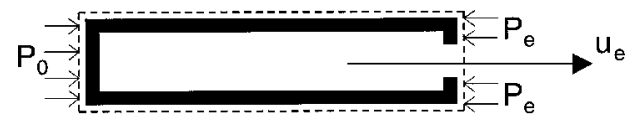

a)

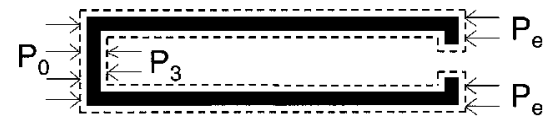

b)

Fig. 9 Control volumes a) typically used in rocket engine analysis and b) used in our analysis.

We assume direct instantaneous initiation of planar detonations at the thrust surface. The effect of indirect initiation is discussed by Cooper et al. ${ }^{9}$ The model assumes that a reflected expansion wave is generated when the detonation wave reaches the open end, which is generally true, as discussed earlier. The model is based on analytical calculations, except for the modeling of the pressure decay period, which results from dimensional analysis and experimental observations.

\section{Determination of the Impulse}

Under our model assumptions, the single-cycleimpulse is generated by the pressure differential at the thrust surface. A typical experimental pressure history at the thrust surface recorded by Cooper et al. ${ }^{9}$ is given in Fig. 10. When the detonation is initiated, the CJ pressure peak is observed before the pressure decreases to $P_{3}$ by the passage of the Taylor wave. ${ }^{17}$ The pressure at the thrust surface remains approximately constant until the first reflected characteristic reaches the thrust surface and the reflected expansion wave decreases the pressure. The pressure is decreased below atmospheric for a period of time before ultimately reaching the atmospheric value (Fig. 8).

For our modeling, the pressure-time trace at the thrust surface has been idealized (Fig. 11). The CJ pressure peak is considered to occur during a negligibly short time. The pressure stays constant for a total time $t_{1}+t_{2}$ at pressure $P_{3}$. Then the pressure is affected by the reflected expansion and eventually decreases to the atmospheric value.

By the use of the control volume defined in Fig. 9b, the singlecycle impulse can be computed as

$$
I=A \int_{0}^{\infty} \Delta P(t) \mathrm{d} t
$$

where ignition is assumed to occur at $t=0$. From the idealized pressure-time trace, the impulse can be decomposedinto three terms

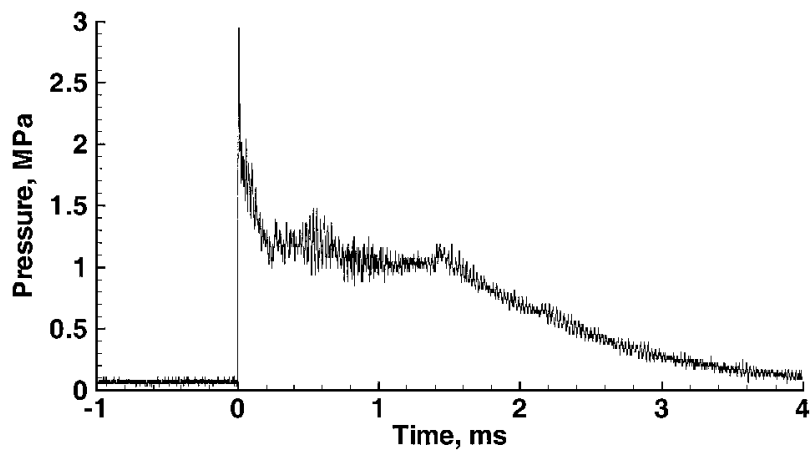

Fig. 10 Sample pressure recorded at the thrust surface ${ }^{9}$ for a mixture of stoichiometric ethylene-oxygen at 1-bar and 300-K initial conditions.

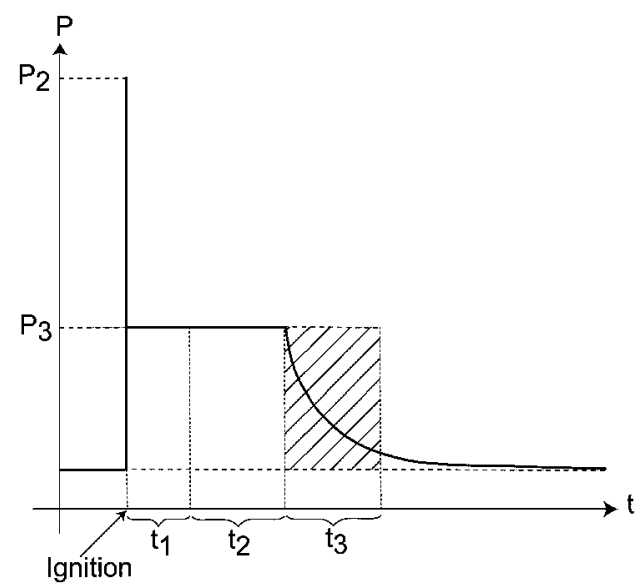

Fig. 11 Idealized model of the thrust surface pressure history.

$$
I=A\left[\Delta P_{3} t_{1}+\Delta P_{3} t_{2}+\int_{t_{1}+t_{2}}^{\infty} \Delta P(t) \mathrm{d} t\right]
$$

The first term on the right-hand side of Eq. (3) represents the contribution to the impulse associated with the detonation propagation during time $t_{1}=L / U_{\mathrm{CJ}}$, the second term is the contribution associated with the time $t_{2}$ required for expansion wave propagation from the open end to the thrust surface, and the third term is associated with the pressure decay period.

The time $t_{2}$ depends primarily on the length of the tube and the characteristic sound speed $c_{3}$ behind the expansion wave, which 
suggests the introduction of a nondimensional parameter $\alpha$, defined by

$$
t_{2}=\alpha L / c_{3}
$$

Dimensional analysis will be used to model the third term on the right-hand side of Eq. (3). The inviscid, compressible flow equations can always be nondimensionalizedusing referenceparameters, which are a sound speed, a characteristic length, and a reference pressure. Thus, we nondimensionalizeour pressure integral in terms of $c_{3}, L$, and $P_{3}$

$$
\int_{t_{1}+t_{2}}^{\infty} \Delta P(t) \mathrm{d} t=\frac{\Delta P_{3} L}{c_{3}} \int_{\tau_{1}+\tau_{2}}^{\infty} \Pi(\tau) \mathrm{d} \tau
$$

The nondimensional integral on the right-hand side of Eq. (5) can depend only on the remaining nondimensional parameters of the flow, which are the ratio of specific heats in the products $\gamma$, the pressure ratio between the constant pressure region and the initial pressure $P_{3} / P_{1}$, and the nondimensional energy release during the detonation process $q / R T_{1}$. We will define the value of this integral to be $\beta$, which has a definite value for a given mixture

$$
\beta\left(\gamma, \frac{P_{3}}{P_{1}}, \frac{q}{R T_{1}}\right)=\int_{\tau_{1}+\tau_{2}}^{\infty} \Pi(\tau) \mathrm{d} \tau
$$

For fuel-air detonations over a limited range of compositions close to stoichiometric, the parameters in Eq. (6) vary by only a modest amount, and we will assume that $\beta$ is approximately constant. This assumption is not crucial in our model, and a more realistic expression for $\beta$ can readily be obtained by numerical simulation. For the present purposes, this assumption is justified by the comparisons with the experimental data shown subsequently.

The dimensional integral on the left-hand side of Eq. (5) can be used to define a characteristic time $t_{3}$, which is related to $\beta$

$$
\int_{t_{1}+t_{2}}^{\infty} \Delta P(t) \mathrm{d} t=\Delta P_{3} t_{3}=\Delta P_{3} \beta \frac{L}{c_{3}}
$$

In Fig. 11, the time $t_{3}$ can be interpreted as the width of the hatched zone representing the equivalent area under the decaying part of the pressure-time trace for $t>t_{1}+t_{2}$. The impulse of Eq. (3) can now be rewritten to include the nondimensional parameters $\alpha$ and $\beta$

$$
I=A \Delta P_{3}\left[L / U_{\mathrm{CJ}}+(\alpha+\beta)\left(L / c_{3}\right)\right]
$$

\section{Determination of $\alpha$}

We have determined $\alpha$ by considering the interaction of the reflected wave and the Taylor wave. ${ }^{17}$ The method of characteristicsis used to derive a similarity solution for the leading characteristic of the reflected expansion. This technique will also work for reflected compressions as long as the waves are sufficiently weak.

The derivation of the expression for $\alpha$ begins by considering the network of characteristicswithin the Taylor wave, ${ }^{17}$ shown in Fig. 4. The Riemann invariant $J^{-}$is conserved along a $C^{-}$characteristic going through the Taylor wave,

$$
J^{-}=u_{2}-2 c_{2} /(\gamma-1)=-2 c_{3} /(\gamma-1)=u-2 c /(\gamma-1)
$$

Inside the Taylor wave, the $C^{+}$characteristics are straight lines with a slope given by $x / t=u+c$. Using the Riemann invariant $J^{-}$to relate $u$ and $c$ to the flow parameters in state 2, we find that

$$
\begin{aligned}
x / c_{2} t & =(u+c) / c_{2} \\
& =u_{2} / c_{2}+[(\gamma+1) /(\gamma-1)]\left(c / c_{2}\right)-2 /(\gamma-1)
\end{aligned}
$$

In particular, this method can be used to derive the flow properties in the Taylor wave. The speed of sound is

$$
c / c_{3}=2 /(\gamma+1)+[(\gamma-1) /(\gamma+1)]\left(x / c_{3} t\right)
$$

where $c_{3}$ is calculated from

$$
c_{3}=c_{2}-[(\gamma-1) / 2] u_{2}=[(\gamma+1) / 2] c_{2}-[(\gamma-1) / 2] U_{\mathrm{CJ}}
$$

Equation(11) is valid in the expansion wave, for $c_{3} t \leq x \leq U_{\mathrm{CJ}} t$. The pressure in the Taylor wave ${ }^{17}$ can be computed using the isentropic flow relations,

$$
P=P_{3}\left\{1-[(\gamma-1) /(\gamma+1)]\left(1-x / c_{3} t\right)\right\}^{2 \gamma /(\gamma-1)}
$$

When the interaction of the reflected expansion wave with the Taylor wave is considered, the slope of the first reflected characteristic $\hat{C}^{-}$can be calculated as

$$
\frac{\mathrm{d} x}{\mathrm{~d} t}=u-c=\frac{x}{t}-2 c
$$

Substituting for $x / t$ from Eq. (10), we find that

$$
\frac{1}{c_{2}} \frac{\mathrm{d} x}{\mathrm{~d} t}+\frac{2(\gamma-1)}{\gamma+1}\left[\frac{u_{2}}{c_{2}}-\frac{2}{\gamma-1}+\frac{3-\gamma}{2(\gamma-1)} \frac{x}{c_{2} t}\right]=0
$$

The form of Eq. (15) suggests the introduction of a similarity variable $\eta=x / c_{2} t$. Making the change of variables, we obtain an ordinary differential equation for $\eta$

$$
t \frac{\mathrm{d} \eta}{\mathrm{d} t}+\frac{2(\gamma-1)}{\gamma+1}\left[\eta-\frac{u_{2}}{c_{2}}+\frac{2}{\gamma-1}\right]=0
$$

The solution to this equation is

$$
\begin{aligned}
\eta(t) & =u_{2} / c_{2}-2 /(\gamma-1) \\
& +[(\gamma+1) /(\gamma-1)]\left(L / U_{\mathrm{CJ}} t\right)^{2(\gamma-1) /(\gamma+1)}
\end{aligned}
$$

where we have used the initial condition $\eta\left(t_{1}\right)=U_{\mathrm{CJ}} / c_{2}$. The last characteristic of the Taylor wave ${ }^{17}$ has a slope $x / t=c_{3}$. Hence, the first reflected characteristic exits the Taylor wave at time $t^{*}$ determined by $\eta\left(t^{*}\right)=c_{3} / c_{2}$. Solving for $t^{*}$, we have

$$
t^{*}=\frac{L}{U_{\mathrm{CJ}}}\left[\left(\frac{\gamma-1}{\gamma+1}\right)\left(\frac{c_{3}-u_{2}}{c_{2}}+\frac{2}{\gamma-1}\right)\right]^{-(\gamma+1) / 2(\gamma-1)}
$$

For $t^{*}<t<t_{1}+t_{2}$, the characteristic $\hat{C}^{-}$propagates at constant velocity equal to the sound speed $c_{3}$. From the geometry of the characteristic network shown in Fig. $4, \hat{C}^{-}$reaches the thrust surface at time $t_{1}+t_{2}=2 t^{*}$. Thus, $t_{2}=2 t^{*}-t_{1}=\alpha L / c_{3}$. Solving for $\alpha$, we obtain

$$
\alpha=\frac{c_{3}}{U_{\mathrm{CJ}}}\left[2\left(\frac{\gamma-1}{\gamma+1} \frac{c_{3}-u_{2}}{c_{2}}+\frac{2}{\gamma-1}\right)^{-(\gamma+1) / 2(\gamma-1)}-1\right]
$$

The quantities involved in this expression essentially depend on two nondimensionalparameters: $\gamma$ and the detonation Mach number $M_{\mathrm{CJ}}=U_{\mathrm{CJ}} / c_{1}$. These can either be computed numerically with realistic thermochemistry or else analytically using the ideal gas one- $\gamma$ model for a CJ detonation. Numerical evaluations of this expression for typical fuel-air detonations show that $\alpha \approx 1.1$ for a wide range of fuel and compositions. When the one- $\gamma$ model is used, the resulting expression for $\alpha\left(\gamma, M_{\mathrm{CJ}}\right)$ is

$$
\begin{aligned}
\frac{1}{2}\left(1+\frac{1}{M_{\mathrm{CJ}}^{2}}\right)\left\{2 \left[\frac { \gamma - 1 } { \gamma + 1 } \left(\frac{\gamma+3}{2}+\frac{2}{\gamma-1}\right.\right.\right. & -1\}
\end{aligned}
$$




\section{Determination of $\boldsymbol{\beta}$}

The region between the first reflected characteristic and the contact surface in Fig. 4 is a nonsimple region created by the interaction of the reflected expansion wave with the Taylor wave. ${ }^{17}$ The multidimensional flow behind the diffracting shock front also plays a significant role in determining the pressure in this region. For these reasons, it is impossible to derive an analytical solution for the parameter $\beta$. It is, however, possible to use experimental data and Eq. (6) to calculate $\beta$. We considered data from Zitoun and Desbordes, ${ }^{8}$ who carried out detonation tube experiments and measured impulse using tubes of different lengths. They showed that the impulse scales with the length of the tube, as expected from Eq. (8).

Zitoun and Desbordes ${ }^{8}$ used an exploding wire to initiate detonations directly, which is representative of the idealized conditions of our model. They determined impulse for stoichiometric ethyleneoxygen mixtures by integrating the pressure differential at the thrust surface. The analysis of their pressure-time traces reveals that the overpressure, after being roughly constant for a certain period of time, decreases and becomes negative before returning to zero. The integration of the decaying part of the pressure-time trace was carried out up to a time late enough (typically greater than $20 t_{1}$ ) to ensure that the overpressure has returned to zero. This integration gave a value of $\beta=0.53$.

\section{Validation of the Model}

The model was validated against experimental data and comparisons were made in terms of impulse per unit volume and specific impulse. The impulse per unit volume is defined as

$$
I_{V}=I / V
$$

The mixture-based specific impulse $I_{\mathrm{sp}}$ is defined as

$$
I_{\text {sp }}=I / \rho_{1} V g=I_{V} / \rho_{1} g=I / M g
$$

The fuel-based specific impulse $I_{\text {spf }}$ is defined with respect to the fuel mass instead of the mixture mass

$$
I_{\mathrm{spf}}=I / \rho_{1} X_{F} V g=I_{\mathrm{sp}} / X_{F}=I / M_{f} g
$$

\section{Comparisons with Single-Cycle Experiments}

The calculation of the parameter $\alpha$ was validated by comparing the arrival time of the reflected expansion wave from experimental pressure histories at the thrust surface with the time calculated from the similarity solution. For a mixture of stoichiometric ethylene-air at 1-barinitial pressure, the time in an experimental pressurehistory ${ }^{9}$ between detonation initiation and the arrival of the reflected expansion wave was $1.43 \mathrm{~ms}$ from a $1.016-\mathrm{m}$ long tube. The corresponding calculated time was $1.39 \mathrm{~ms}$, within $3 \%$ of the experimental value. Similarly, comparison with data ${ }^{8}$ for a tube of length $0.225 \mathrm{~m}$ showed that excellent agreement (within 1\%) is obtained between our calculated value $(313 \mu \mathrm{s})$ and experiment $(315 \mu \mathrm{s})$.

The value of $\beta$ was also computed using data from our experiments $^{9}$ with stoichiometric ethylene-oxygen. Because these experiments used deflagration-to-detonation transition (DDT), we were able to compare with only two cases using an unobstructed tube and an initial pressure of 1 bar for which there was very rapid onset of detonation. These cases correspond to values of $\beta$ equal to 0.55 and 0.66 . Note that these values are sensitive to the time at which the integration is started. We computed this time using our theoretical values of $t_{1}$ and $t_{2}$.

Model predictions of impulse per unit volume were compared with data from Cooper et al. ${ }^{9}$ Direct experimental impulse measurements were obtained with a ballistic pendulum, and detonation initiation was obtained via DDT. Obstacles were mounted inside the detonation tube in some of the experiments to enhance DDT. A correlation plot showing the impulse per unit volume obtained with the model vs the experimental values is displayed in Fig. 12. The values displayed here cover experiments with four different fuels (hydrogen, acetylene, ethylene, and propane) over a range of initial conditions and compositions. The solid line represents perfect

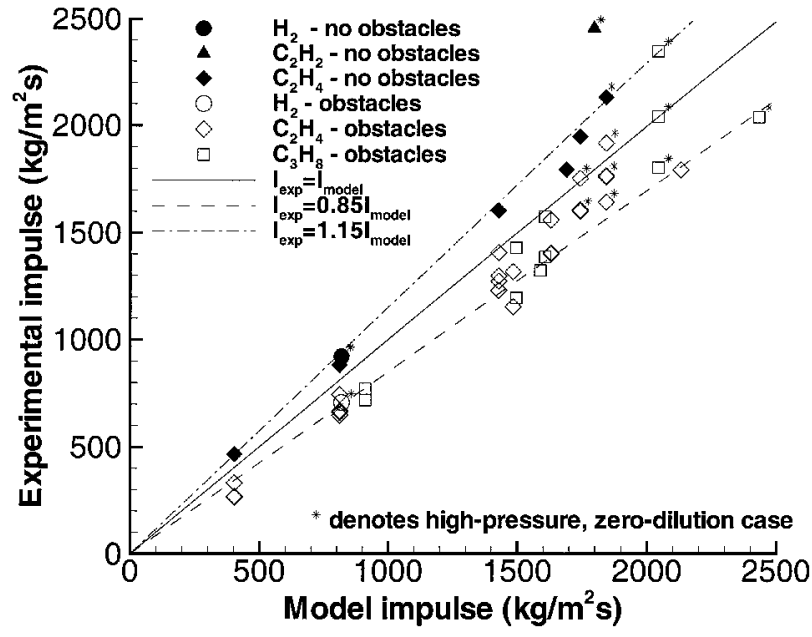

Fig. 12 Model predictions vs experimental data for the impulse per unit volume: $\bullet, \Delta$, and $\downarrow$ represent data for unobstructed tubes, whereas $\circ, \diamond$, and $\square$ show data for cases in which obstacles were used; lines corresponding to $+\mathbf{1 5 \%}$ and $-\mathbf{1 5 \%}$ deviation from the model values are also shown; and $*$ denote high-pressure (higher than 0.8 bar), zero-dilution cases.

correlation between the experimentaldata and the model. The filled symbols represent the data for unobstructed tubes, whereas the open symbols correspond to cases for which obstacles were used in the detonation tube.

The analytical model predictions were close to the experimental values of the impulse as shown in Fig. 12. The model assumes direct initiation of detonation, and so it does not take into account any DDT phenomenon. The agreement is better for cases with high initial pressure and no nitrogen dilution because the DDT time (time it takes the initial flame to transition to a detonation) is the shortest for these mixtures. For the unobstructed tube experiments, the model systematically underpredicts the impulse by 5 to $15 \%$, except for the acetylene case, where it is about $25 \%$ too low. When obstacles are used, the experimental values are up to $25 \%$ lower than the model predictions. In general, the discrepancy between model and experiment is less than or equal to $\pm 15 \%$. This conclusion is supported in Fig. 12 by the $\pm 15 \%$ deviation lines that encompass the experimental data. The lower experimental values for cases with obstacles are apparently caused by the additional form drag associated with the separated flow over the obstacles.

The model parameters are relatively constant, $1.07<\alpha<1.13$ and $0.53<\beta<0.66$, for all of the mixtures studied here. A reasonable estimate for $\alpha$ is 1.1 and for $\beta$ is 0.53 . The ratio $U_{\mathrm{CJ}} / c_{3}$ for fuel-oxygen-nitrogen mixtures is approximately 2 . For quick estimates of the impulse, these values can be used in Eq. (8) to obtain the approximate model prediction formula

$$
I=4.3\left(\Delta P_{3} / U_{\mathrm{CJ}}\right) A L=4.3\left(\Delta P_{3} / U_{\mathrm{CJ}}\right) V
$$

The approximate formula reproduces the exact expressions within $2.5 \%$.

Zitoun and Desbordes ${ }^{8}$ calculated the single-cycle specific impulse for various reactive mixtures based on a formula developed from their experimental data for ethylene-oxygen mixtures: $I_{\text {sp }}=K \Delta P_{3} /\left(g \rho_{1} U_{\mathrm{CJ}}\right)$. The coefficient $K$ is estimated to be 5.4 in their study, whereas we obtained an estimate of 4.3. This accounts for the differencein the specific impulse results presented in Table 1. The present analytical model impulse is about $20 \%$ lower than Zitoun and Desbordes's ${ }^{8}$ predictions. This difference can be explained by the fact that Zitoun and Desbordes ${ }^{8}$ considered only the region of positive overpressure, which extends to about $9 t_{1}$, in their integration of the pressure differential. They based this on the assumption that the following region of negative overpressure would be used for the self-aspiration of air in a multicycle airbreathing application. However, because we were interested in comparing with ballistic pendulum measurements, we performed the integration 
Table 1 Comparison of the model predictions for the mixture-based specific impulse

\begin{tabular}{lcc}
\hline \hline Mixture & Model $I_{\text {sp }}$ & Zitoun and Desbordes ${ }^{8}$ \\
\hline $\mathrm{C}_{2} \mathrm{H}_{4}+3 \mathrm{O}_{2}$ & 151.1 & 200 \\
$\mathrm{C}_{2} \mathrm{H}_{4}+3\left(\mathrm{O}_{2}+3.76 \mathrm{~N}_{2}\right)$ & 117.3 & 142 \\
$\mathrm{C}_{2} \mathrm{H}_{2}+2.5 \mathrm{O}_{2}$ & 150.9 & 203 \\
$\mathrm{C}_{2} \mathrm{H}_{2}+2.5\left(\mathrm{O}_{2}+3.76 \mathrm{~N}_{2}\right)$ & 120.6 & 147 \\
$\mathrm{H}_{2}+0.5 \mathrm{O}_{2}$ & 172.9 & 226 \\
$\mathrm{H}_{2}+0.5\left(\mathrm{O}_{2}+3.76 \mathrm{~N}_{2}\right)$ & 123.7 & 149 \\
\hline \hline
\end{tabular}

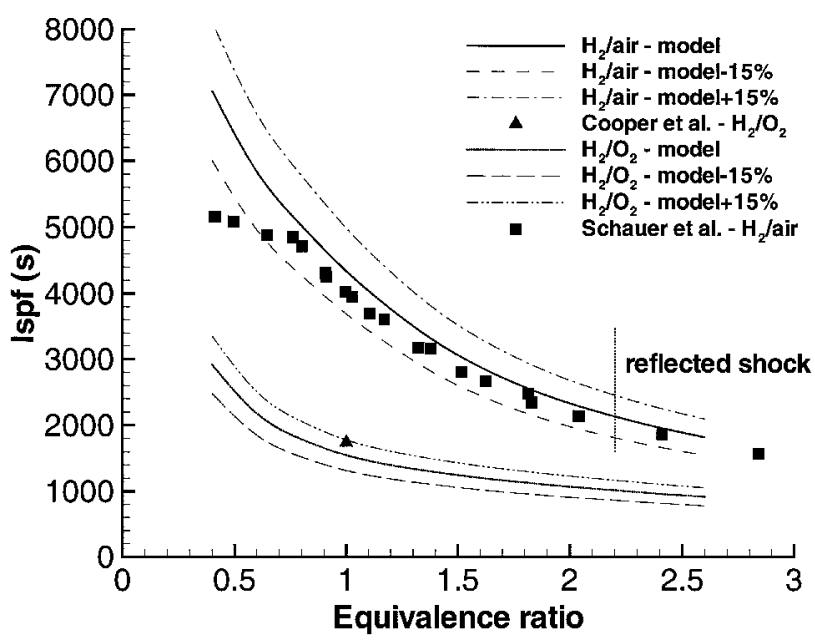

Fig. 13 Comparison of specific impulse between model predictions and experimental data for hydrogen-air ${ }^{23}$ with varying equivalence ratio and stoichiometric hydrogen-oxygen ${ }^{9}$; nominal initial conditions are $P_{1}=1$ bar and $T_{1}=300 \mathrm{~K}$, and lines corresponding to $+15 \%$ and $-15 \%$ deviation from the model values are also shown.

until the overpressure was back to zero, which occurs at about $20 t_{1}$. The region of negative overpressure between 9 and $20 t_{1}$ results in an impulse decrease. If we calculate the value of $\beta$ by limiting the integration to the time of positive overpressure, we obtain a value of $K=4.8$

\section{Comparisons with Multicycle Experiments}

Calculations of specific impulse and thrust were compared to experimental data from Schauer et al. ${ }^{23,24}$ Their facility consisted of a 50.8-mm-diam by 914.4-mm long tube mounted on a damped thrust stand. Impulse and thrust measurements were made in hydrogenair $^{23}$ and propane-air ${ }^{24}$ mixtures with varying equivalence ratio. Data were collected during continuous multicycle operation, and the thrust was averaged over many cycles. To compare with our model predictions, we assume multicycle operation is equivalent to a sequence of ideal single cycles. In multicycle operation, a portion of the cycle time is used to purge the tube and refill with reactants. The expulsion of gas from the tube can result in a contribution to the impulse that is not accounted for in our simple model. To estimate the magnitude of the impulse during refilling, we assumed that the detonation and exhaust phase had a duration of about $10 t_{1}$ and that the remaining portion of the cycle is used for the purging and filling processes. We found that the contribution of the purge and fill portion to the thrust was less than the Schauer et al. stated experimental uncertainty of $6 \%$ (Ref. 23).

Comparisons of specific impulse are presented in Fig. 13 for hydrogen-air ${ }^{23}$ and in Fig. 14 for propane-air. ${ }^{24}$ For comparison, predictions and one single-cycle measurement for hydrogenoxygen are shown in Fig. 13. Two sets of data are shown for propane: data labeled det are from runs in which the average detonation wave velocity was about $80 \%$ of the CJ value, and data labeled no det? are from runs in which detonations were unstable or intermittent. The impulse model predictions are within $8 \%$ of the experimental data for hydrogen-air at $\phi>0.8$, and within $15 \%$ for stable propane-air cases. Figure 13 also includes an experimental hydrogen-oxygen single-cycle data point from our own experiments. 'The vertical

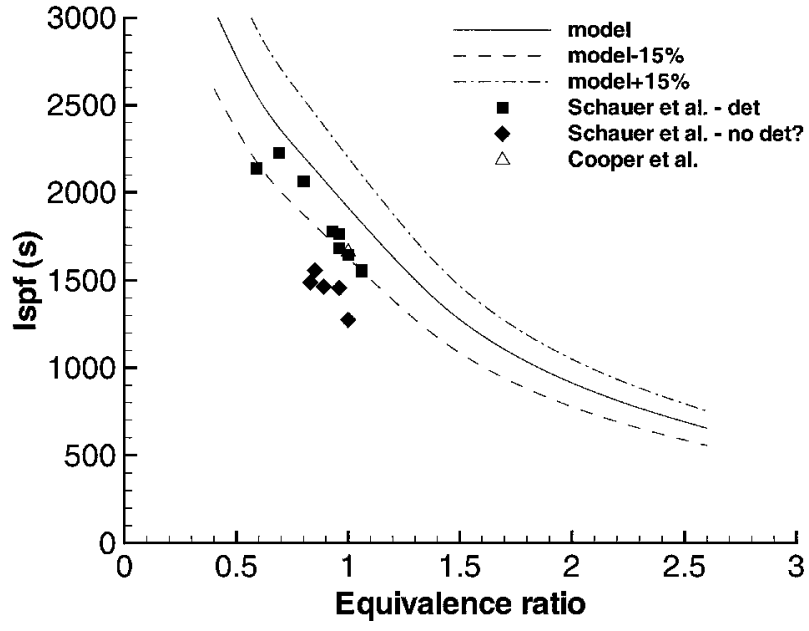

Fig. 14 Comparison of specific impulse between model predictions and experimental data ${ }^{9,24}$ for propane-air with varying equivalence ratio; nominal initial conditions are $P_{1}=1$ bar and $T_{1}=300 \mathrm{~K}$, and lines corresponding to $+15 \%$ and $-15 \%$ deviation from the model values are also shown.

dashed line on Fig. 13 denotes a limit of the model validity. For richer mixtures, a reflected shock is calculated (Figs. 2 and 3). That the model still correctly predicts the impulse beyond this limit suggests that the reflected shock is weak and does not significantly affect the integrated pressure. Indeed, a ballistic pendulum experiment ${ }^{9}$ carried out with hydrogen-oxygen resulted in the directly measured impulse being within $10 \%$ of the value predicted by the model (Fig. 13). Figures 13 and 14 also include $\pm 15 \%$ deviation lines from the model predictions.

In Fig. 14, the significantly lower impulse of the experimental point at $\phi=0.59$ in propane mixtures is certainly due to cell size effects. At the lower equivalenceratios, the cell size $\mathrm{e}^{25}$ of propane-air ( $152 \mathrm{~mm}$ at $\phi=0.74$ ) approaches $\pi$ times the diameter of the tube, which is the nominal limit for stable detonation propagation. ${ }^{26,27}$

In the case of hydrogen-air (Fig. 13), the cell size ${ }^{25}$ at $\phi=0.75$ is $21 \mathrm{~mm}$, so that the decrease in the experimental impulse data at low equivalence ratios can not be explained by cell size effects. Following the work of Dorofeev et al., ${ }^{28}$ the magnitude of the expansion ratio was examined for these mixtures. However, calculations for lean hydrogen-air showed that the expansion ratio is always higher than the critical value defined ${ }^{28}$ for hydrogen mixtures. Instead, the results may be explained by the transition distance of the mixtures. Dorofeev et al. ${ }^{29}$ studied the effect of scale on the onset of detonations. They proposed and validated a criterion for successful transition to detonation: $\mathcal{L}>7 \lambda$, where $\mathcal{L}$ is the characteristic geometrical size (defined to account for the presence of obstacles) and $\lambda$ the cell size of the mixture. Schauer et al. ${ }^{23}$ used a $45.7-\mathrm{mm}$ pitch Shchelkin spiral constructed of $4.8-\mathrm{mm}$-diam wire to initiate detonations in their detonation tube. As defined by Dorofeev, ${ }^{29}$ this results in a characteristic geometrical size of $257 \mathrm{~mm}$, comparable to $7 \lambda=217 \mathrm{~mm}$ for a value of $\phi=0.67$. The cell size increases with decreasing equivalenceratio for lean mixtures, and so mixtures with equivalence ratios smaller than 0.67 will not transition to detonation within the spiral or possibly even the tube itself. This is consistent with the data shown in Fig. 13; hydrogen-air tests with $\phi \leq 0.67$ have experimental specific impulse values significantly lower than the model prediction. Similar reductions in $I_{\mathrm{sp}}$ were also observed by Cooper et al. ${ }^{9}$ in single-cycle tests of propane-oxygen-nitrogen and ethylene-oxygen-nitrogen mixtures with greater than a critical amount of nitrogen dilution.

Average thrust for multicycle operation can be calculated from our single-cycle impulse model predictions, assuming a periodic sequence of individual pulses that do not interact. For a given singlecycle performance and tube size, the average thrust is proportional to the frequency $f$

$$
T=I_{V} V f
$$




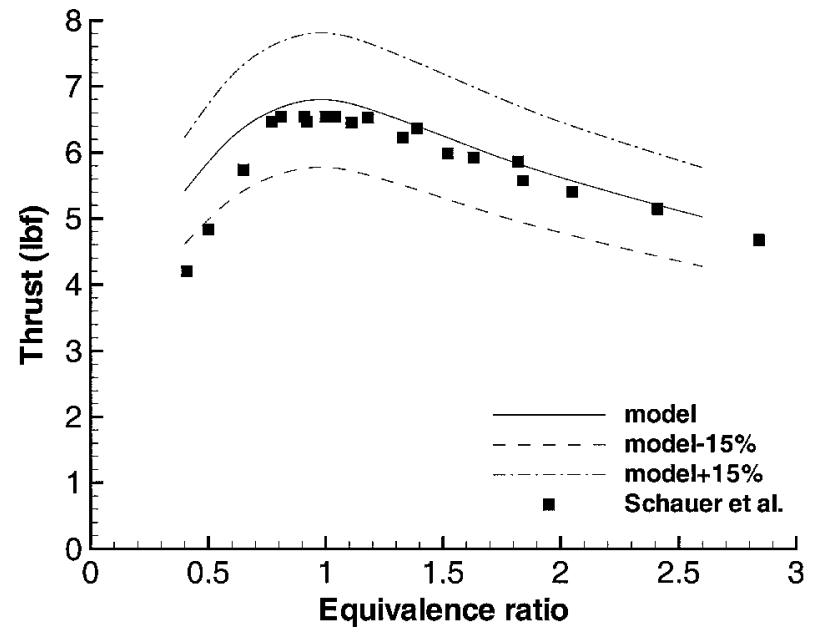

Fig. 15 Thrust prediction for a 50.8-mm diameter by $914.4 \mathrm{~mm}$ long hydrogen-air PDE operated at $16 \mathrm{~Hz}$ (comparison with experimental data of Schauer et al. ${ }^{23}$ ); nominal initial conditions are $P_{1}=1$ bar and $T_{1}=300 \mathrm{~K}$, and lines corresponding to $+15 \%$ and $-15 \%$ deviation from the model values are also shown.

Schauer et al. ${ }^{23}$ measured the average thrust in multicycle operation with hydrogen-air over a range of frequencies between 14 and $40 \mathrm{~Hz}$ and verified the linear dependence on frequency. Although this simple model suggests that thrust can be increased indefinitely by increasing the cycle frequency, there are obvious physical constraints ${ }^{30}$ that limit the maximum frequency for given size tube. The maximum cycle frequency is inversely proportional to the sum of the minimum detonation, exhaust, fill, and purge times. The purge and fill times are typically much longer than the detonation and exhaust time and, therefore, are the limiting factors in determining the maximum cycle frequency.

Figure 15 compares measurements ${ }^{23}$ and model predictions for operation at a fixed frequency of $16 \mathrm{~Hz}$. The computation of the thrust with the model is within $4 \%$ of the experimental data for $\phi>0.8$. The discrepancies at low equivalence ratios are due to the increased transition distance discussed earlier.

\section{Comparisons with Numerical Simulations}

Data from the numerical simulation presented earlier in this paper were used to compute the impulse per unit volume. The pressure at the thrust surface (Fig. 8) was integrated over time to obtain the impulse per unit area. Because the simulation was carried out for nonreactive flow and started as the detonation front exited the tube, the initial time corresponding to the detonation travel from the closed end to the open end of the tube was not simulated, but was taken to be $L / U_{\mathrm{CJ}}$. The integration was performed up to a time corresponding to $20 t_{1}$, and the impulse per unit volume was

$$
I / V=22.6\left(P_{1} / U_{\mathrm{CJ}}\right)
$$

This result is within $0.1 \%$ of the approximate model formula of Eq. (24). The simulation results are valid only for cases where the initial pressure $P_{1}$ is equal to the pressure outside the detonation tube $P_{0}$.

Comparisons with numerical computations of specific impulse by other researchers can also be made. Numerical simulations are very sensitive to the specification of the outflow boundary conditionat the open end, and the numerical results vary widely when different types of boundary conditions are used. Sterling et al. ${ }^{1}$ obtained an average value of $5151 \mathrm{~s}$ for the fuel-based specific impulse of a stoichiometric hydrogen-air mixture in a multicycle simulation using a constant pressure boundary condition. Bussing et al. ${ }^{3}$ obtained a range of values of 7500-8000 s. Other predictions by Cambier and Tegner, ${ }^{4}$ including a correction for the effect of the initiation process, gave values between 3000 and 3800 s. More recently, Kailasanath and Patnaik ${ }^{5}$ tried to reconcile these different studies for hydrogenair by highlighting the effect of the outflow boundary condition.
They varied the pressure relaxation rate at the exit and obtained a range of values from 4850 (constant pressure case) to $7930 \mathrm{~s}$ (gradual relaxation case). Our analytical model predicts $4335 \mathrm{~s}$ for the fuel-based specific impulse of stoichiometric hydrogen-air and the experimental value of Schauer et al. ${ }^{23}$ is $4024 \mathrm{~s}$.

\section{Impulse Scaling Relationships}

From Eq. (24), the impulse can be written as

$$
I=K \cdot V\left(\Delta P_{3} / U_{\mathrm{CJ}}\right)
$$

where $K$ has a weak dependence on the properties of the mixture, $K\left(\gamma, q / R T_{1}\right)$. For the purposes of predicting how the impulse depends on the mixture properties and tube size, the principal dependencies are explicitly given in Eq. (27) with $K=$ constant. The dependence of impulse on the mixture properties comes in through the thermodynamic quantities $U_{\mathrm{CJ}}$ and $\Delta P_{3}$. The CJ velocity is a function of composition only and is independent of initial pressure as long as it is not so low that dissociation of the detonation products is significant. For the case of $P_{1}=P_{0}$, the impulse can be written

$$
I=K\left(V P_{1} / U_{\mathrm{CJ}}\right)\left[\left(P_{2} / P_{1}\right)\left(P_{3} / P_{2}\right)-1\right]
$$

From the gasdynamic considerationsgiven in the preceding section, Eq. (13) implies that

$$
P_{3} / P_{2}=\left\{1-[(\gamma-1) /(\gamma+1)]\left(1-U_{\mathrm{CJ}} / c_{3}\right)\right\}^{-2 \gamma /(\gamma-1)}
$$

Equilibrium computations with realistic thermochemistry indicate that $U_{\mathrm{CJ}} / c_{3} \approx 2$ and $0.324 \leq P_{3} / P_{2} \leq 0.375$ with an average value of 0.35 for a wide range of compositions and initial conditions. Under these conditions, the pressure ratio is approximately constant,

$$
P_{3} / P_{2} \approx[2 \gamma /(\gamma+1)]^{-2 \gamma /(\gamma-1)}
$$

The approximate value of Eq. (30) is within 7\% of the exact value of Eq. (29) for a range of mixtures including hydrogen, acetylene, ethylene, propane, and JP10 with air and oxygen and varying nitrogen dilution (0-60\%) at initial conditions $P_{1}=1$ bar and $T_{1}=300 \mathrm{~K}$. This indicates that the impulse will be mainly dependent on the CJ conditions and the total volume of explosive mixture:

$$
I \propto V P_{2} / U_{\mathrm{CJ}}
$$

Values of the CJ parameters and model impulses for several stoichiometric fuel-oxygen-nitrogen mixtures are given in Table 2.

\section{Dependence of Impulse on Energy Content}

To compute the dependence of impulse on energy content, explicitly, the approximate one- $\gamma$ model of a detonation can be used. The CJ Mach number can be written

$$
M_{\mathrm{CJ}}=\sqrt{1+\mathcal{H}}+\sqrt{\mathcal{H}} \quad \text { where } \quad \mathcal{H}=\left[\left(\gamma^{2}-1\right) / 2 \gamma\right]\left(q / R T_{1}\right)
$$

The effective specific energy release $q$ is generally less than the actual specific heat of combustion $q_{c}$ due to the effects of dissociation, specific heat dependence on temperature, and the difference in average molar mass of reactants and products. Values of $\gamma, q_{c}$, and $q$ are given for selected fuel-oxygen-nitrogen mixtures in Table 2, and the computation of $q$ is discussed subsequently. For large values of the parameter $\mathcal{H}$, we can approximate the $\mathrm{CJ}$ velocity as

$$
M_{\mathrm{CJ}} \approx 2 \sqrt{\mathcal{H}} \quad \text { or } \quad U_{\mathrm{CJ}} \approx \sqrt{2\left(\gamma^{2}-1\right) q}
$$

The pressure ratio $\Delta P_{3} / P_{1}$ is also a function of composition, only as long as the initial pressure is sufficiently high. The one- $\gamma$ model can be used to compute the CJ pressure as

$$
\frac{P_{2}}{P_{1}}=\frac{\gamma M_{\mathrm{CJ}}^{2}+1}{\gamma+1}
$$


Table 2 Detonation CJ parameters and computed impulse for selected stoichiometric mixtures at 1-bar initial pressure and 300-K initial temperature

\begin{tabular}{|c|c|c|c|c|c|c|c|c|}
\hline Mixture & $q_{c}, \mathrm{MJ} / \mathrm{kg}$ & $\gamma$ & $P_{2}$, bar & $T_{2}, \mathrm{~K}$ & $U_{\mathrm{CJ}}, \mathrm{m} / \mathrm{s}$ & $M_{\mathrm{CJ}}$ & $I_{\mathrm{sp}}, \mathrm{s}$ & $q, \mathrm{MJ} / \mathrm{kg}$ \\
\hline $\mathrm{H}_{2}-\mathrm{O}_{2}$ & 13.29 & 1.223 & 18.72 & 3679 & 2840 & 5.26 & 172.9 & 6.59 \\
\hline $\mathrm{H}_{2}-\mathrm{O}_{2}-20 \% \mathrm{~N}_{2}$ & 8.39 & 1.189 & 17.98 & 3501 & 2474 & 5.16 & 155.4 & 5.80 \\
\hline $\mathrm{H}_{2}-\mathrm{O}_{2}-40 \% \mathrm{~N}_{2}$ & 5.20 & 1.170 & 16.95 & 3256 & 2187 & 5.01 & 138.7 & 4.98 \\
\hline $\mathrm{H}_{2}$-air & 3.39 & 1.175 & 15.51 & 2948 & 1971 & 4.81 & 123.7 & 3.92 \\
\hline $\mathrm{C}_{2} \mathrm{H}_{2}-\mathrm{O}_{2}$ & 11.82 & 1.263 & 33.63 & 4209 & 2424 & 7.32 & 150.9 & 4.50 \\
\hline $\mathrm{C}_{2} \mathrm{H}_{2}-\mathrm{O}_{2}-20 \% \mathrm{~N}_{2}$ & 9.60 & 1.238 & 30.17 & 4051 & 2311 & 6.89 & 146.0 & 4.37 \\
\hline $\mathrm{C}_{2} \mathrm{H}_{2}-\mathrm{O}_{2}-40 \% \mathrm{~N}_{2}$ & 7.31 & 1.212 & 26.53 & 3836 & 2181 & 6.42 & 139.8 & 4.32 \\
\hline $\mathrm{C}_{2} \mathrm{H}_{2}-\mathrm{O}_{2}-60 \% \mathrm{~N}_{2}$ & 4.95 & 1.186 & 22.46 & 3505 & 2021 & 5.87 & 130.6 & 4.09 \\
\hline $\mathrm{C}_{2} \mathrm{H}_{2}$-air & 3.39 & 1.179 & 19.20 & 3147 & 1879 & 5.42 & 120.6 & 3.60 \\
\hline $\mathrm{C}_{2} \mathrm{H}_{4}-\mathrm{O}_{2}$ & 10.67 & 1.236 & 33.27 & 3935 & 2376 & 7.24 & 151.0 & 4.76 \\
\hline $\mathrm{C}_{2} \mathrm{H}_{4}-\mathrm{O}_{2}-20 \% \mathrm{~N}_{2}$ & 8.70 & 1.210 & 29.57 & 3783 & 2258 & 6.79 & 145.7 & 4.72 \\
\hline $\mathrm{C}_{2} \mathrm{H}_{4}-\mathrm{O}_{2}-40 \% \mathrm{~N}_{2}$ & 6.66 & 1.187 & 25.89 & 3589 & 2132 & 6.32 & 139.1 & 4.60 \\
\hline $\mathrm{C}_{2} \mathrm{H}_{4}-\mathrm{O}_{2}-60 \% \mathrm{~N}_{2}$ & 4.53 & 1.169 & 21.82 & 3291 & 1977 & 5.77 & 129.3 & 4.26 \\
\hline $\mathrm{C}_{2} \mathrm{H}_{4}$-air & 3.01 & 1.172 & 18.25 & 2926 & 1825 & 5.27 & 117.0 & 3.51 \\
\hline $\mathrm{C}_{3} \mathrm{H}_{8}-\mathrm{O}_{2}$ & 10.04 & 1.220 & 36.04 & 3826 & 2360 & 7.67 & 152.7 & 5.20 \\
\hline $\mathrm{C}_{3} \mathrm{H}_{8}-\mathrm{O}_{2}-20 \% \mathrm{~N}_{2}$ & 8.33 & 1.199 & 31.73 & 3688 & 2251 & 7.14 & 147.3 & 5.10 \\
\hline $\mathrm{C}_{3} \mathrm{H}_{8}-\mathrm{O}_{2}-40 \% \mathrm{~N}_{2}$ & 6.48 & 1.181 & 27.45 & 3513 & 2131 & 6.58 & 140.4 & 4.90 \\
\hline $\mathrm{C}_{3} \mathrm{H}_{8}-\mathrm{O}_{2}-60 \% \mathrm{~N}_{2}$ & 4.49 & 1.166 & 22.79 & 3239 & 1980 & 5.95 & 130.3 & 4.45 \\
\hline $\mathrm{C}_{3} \mathrm{H}_{8}$-air & 2.80 & 1.174 & 18.15 & 2823 & 1801 & 5.29 & 115.4 & 3.41 \\
\hline $\mathrm{JP} 10-\mathrm{O}_{2}$ & 9.83 & 1.226 & 38.89 & 3899 & 2294 & 7.99 & 148.4 & 4.84 \\
\hline $\mathrm{JP} 10-\mathrm{O}_{2}-20 \% \mathrm{~N}_{2}$ & 8.34 & 1.205 & 34.00 & 3759 & 2204 & 7.41 & 144.1 & 4.80 \\
\hline $\mathrm{JP} 10-\mathrm{O}_{2}-40 \% \mathrm{~N}_{2}$ & 6.65 & 1.186 & 29.18 & 3585 & 2103 & 6.81 & 138.5 & 4.67 \\
\hline $\mathrm{JP} 10-\mathrm{O}_{2}-60 \% \mathrm{~N}_{2}$ & 4.73 & 1.169 & 24.06 & 3316 & 1972 & 6.12 & 130.1 & 4.37 \\
\hline JP10-air & 2.79 & 1.173 & 18.40 & 2843 & 1784 & 5.32 & 114.6 & 3.38 \\
\hline
\end{tabular}

For large values of the parameter $\mathcal{H}$, equivalent to large $M_{\mathrm{CJ}}$, this can be approximated as

$$
P_{2} \approx[1 /(\gamma+1)] \rho_{1} U_{\mathrm{CJ}}^{2}
$$

In the same way, we can approximate, assuming $P_{1}=P_{0}$,

$$
\Delta P_{3} / P_{1}=\left(P_{2} / P_{1}\right)\left(P_{3} / P_{2}\right)-1 \approx\left(P_{2} / P_{1}\right)\left(P_{3} / P_{2}\right)
$$

and the impulse can be approximated as

$$
I \approx[1 /(\gamma+1)] M U_{\mathrm{CJ}} K\left(P_{3} / P_{2}\right)
$$

By the use of the approximation of Eq. (33), this can be written

$$
I \approx M \sqrt{q}\left\{\sqrt{2[(\gamma-1) /(\gamma+1)]} K\left(P_{3} / P_{2}\right)\right\}
$$

The term in the braces is only weakly dependent on the mixture composition. With Eq. (30), the impulse can be approximated as

$$
I \approx M \sqrt{q} K \sqrt{2[(\gamma-1) /(\gamma+1)]}[2 \gamma /(\gamma+1)]^{-2 \gamma /(\gamma-1)}
$$

This expression indicates that the impulse is directly proportional to the product of the total mass of explosive mixture in the tube and the square root of the specific energy content of the mixture:

$$
I \propto M \sqrt{q}
$$

\section{Dependence of Impulse on Initial Pressure}

At fixed composition and initial temperature, the values of $q, \gamma$, and $R$ are constant. Equilibrium computations with realistic thermochemistry show that, for high enough initial pressures, $U_{\mathrm{CJ}}, P_{3} / P_{2}$, and $P_{2} / P_{1}$ are essentially independent of initial pressure. From Eq. (39), we conclude that the impulse (or impulse per unit volume) is directly proportional to initial pressure under these conditions because $M=\rho_{1} V=P_{1} V / R T_{1}$ :

$$
I \propto V P_{1}
$$

\section{Dependence of Impulse on Initial Temperature}

At fixed composition and initial pressure, the impulse decreases with increasing initial temperature. This is because the mass in the detonation tube varies inversely with initial temperature when the pressure is fixed. From Eq. (39), we have

$$
I \propto V / T_{1}
$$

\section{Mixture-Based Specific Impulse}

At fixed composition, the mixture-based specific impulse is essentially independent of initial pressure and initial temperature:

$$
\begin{aligned}
I_{\mathrm{sp}}= & I / M g \approx(\sqrt{q} / g) \\
& \times K \sqrt{2[(\gamma-1) /(\gamma+1)]}[2 \gamma /(\gamma+1)]^{-2 \gamma /(\gamma-1)}
\end{aligned}
$$

This also holds for the fuel-based specific impulse because, at fixed composition, the fuel mass is a fixed fraction of the total mass. More generally, Eq. (43) shows that the specific impulse is proportional to the square root of the specific energy content of the explosive mixture,

$$
I_{\mathrm{sp}} \propto \sqrt{q}
$$

The coefficient in Eq. (43) can be numerically evaluated using our value of the coefficient $K$ of 4.3 and a value of $\gamma$ obtained from equilibrium computations. ${ }^{18}$ The range of $\gamma$ for the mixtures considered (Table 2) was $1.16<\gamma<1.26$, where fuel-oxygen-nitrogen mixtures usually have a higher $\gamma$ than undiluted fuel-oxygen mixtures. The resulting coefficient of proportionality in Eq. (44) is between 0.061 and 0.071 , with an average value of 0.065 when $q$ is expressed in Joules per kilogram, so that $I_{\mathrm{sp}} \approx 0.065 \sqrt{q}$.

The value of $q$ is calculated with Eq. (32) and the results (Table 2) of equilibrium computations of $M_{\mathrm{CJ}}$ and $\gamma$. Equation (32) can be rearranged to give $q$ explicitly,

$$
q=\frac{\gamma R T_{1}}{2\left(\gamma^{2}-1\right)}\left(M_{\mathrm{CJ}}-\frac{1}{M_{\mathrm{CJ}}}\right)^{2}
$$

Values of $q$ given in Table 2 were computed using this expression with a gas constant based on the reactant molar mass. Note that the values of $q$ computed in this fashion are significantly less than the specific heat of combustion $q_{c}$ when the CJ temperature is above $3500 \mathrm{~K}$. This is due to dissociation of the major products reducing the temperature and the effective energy release.

The scaling relationship of Eq. (44) is tested in Fig. 16 by plotting the model impulse $I_{\mathrm{sp}}$ vs the effective specific energy release $q$ for all of the cases shown in Table 2. The approximate relationship $I_{\mathrm{sp}} \approx 0.065 \sqrt{q}$ is also shown. In general, higher values of the specific impulse correspond to mixtures with a lower nitrogen dilution and, hence, a higher energy release, for which the CJ temperature is higher and dissociation reactions are favored. There is reasonable 


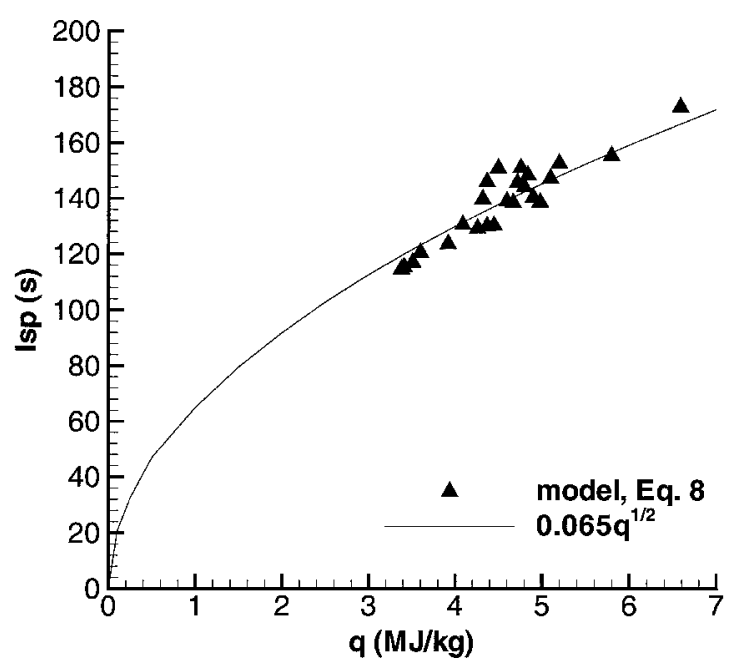

Fig. 16 Specific impulse scaling with energy content; model predictions [Eq. (8)] vs effective specific energy content $q$ for hydrogen, acetylene, ethylene, propane, and JP10 with air and oxygen including $0,20,40$, and $60 \%$ nitrogen dilution at $P_{1}=1$ bar and $T_{1}=300 \mathrm{~K}$.

agreement between the model $I_{\mathrm{sp}}$ and the approximate square root scaling relationshipwith a fixed coefficient of proportionality.However, there is significant scatter about the average trend due to the dependence of $\gamma$ on the mixture composition and temperature. Including this dependence substantially improves the agreement, and the predictions of Eq. (43) are within 3.5\% of the values computed by Eq. (8).

\section{Impulse Predictions: Parametric Studies}

Impulse calculations were carried out for different mixtures, equivalence ratios, initial pressures, and nitrogen dilutions. Unless otherwise mentioned, all calculations were performed with an initial temperature of $300 \mathrm{~K}$.

The model input parameters consist of the external environment pressure $P_{0}$, the detonation velocity $U_{\mathrm{CJ}}$, the sound speed behind the detonation front $c_{2}$, the CJ pressure $P_{2}$, and the ratio of the specific heats of the products $\gamma$. All parameters were computed using equilibrium calculations ${ }^{18}$ performed with a realistic set of combustion products. The input parameters were used in Eqs. (12), (29), and (19) to calculate $P_{3}$ and $\alpha$. The parameters were then used in Eq. (8) to obtain the impulse.

The impulse is calculated for the following fuels: ethylene, propane, acetylene, hydrogen, Jet A, and JP10 with varying initial pressure, equivalence ratio, and nitrogen dilution. Results are expressed in terms of impulse per unit volume of the tube, specific impulse, and fuel-based specific impulse. Results for hydrogenoxygen mixtures are strictly valid for equivalence ratios less than 0.8 and for hydrogen-air mixtures with equivalence ratios less than 2.2. In these cases, the calculations are probably reasonable estimates, but the reader should keep in mind that the underlying physical assumption is no longer justified. The results for Jet A and JP10 assume that these fuels are in completely vaporized form for all initial conditions. Although unrealistic at low temperatures, this gives a uniform basis for comparison of all fuels.

\section{Impulse per Unit Volume}

The impulse per unit volume is independent of the tube size and is linearly dependent on the initial pressure, as indicated by Eq. (41). The variation of $I_{V}$ with $P_{1}, \phi$, and $\mathrm{N}_{2} \%$ is shown in Figs. 17, 18, and 19 . Hydrogen cases are very different from hydrocarbons. The impulse per unit volume is much lower due to the lower molecular mass of hydrogen, which results in lower density and CJ pressure. Equation (40) shows that the impulse per unit volume is proportional to the density of the explosive mixture and the square root of the specific energy release. The specific energy release of hydrogen mixtures is of the same order as that obtained with other fuels, but

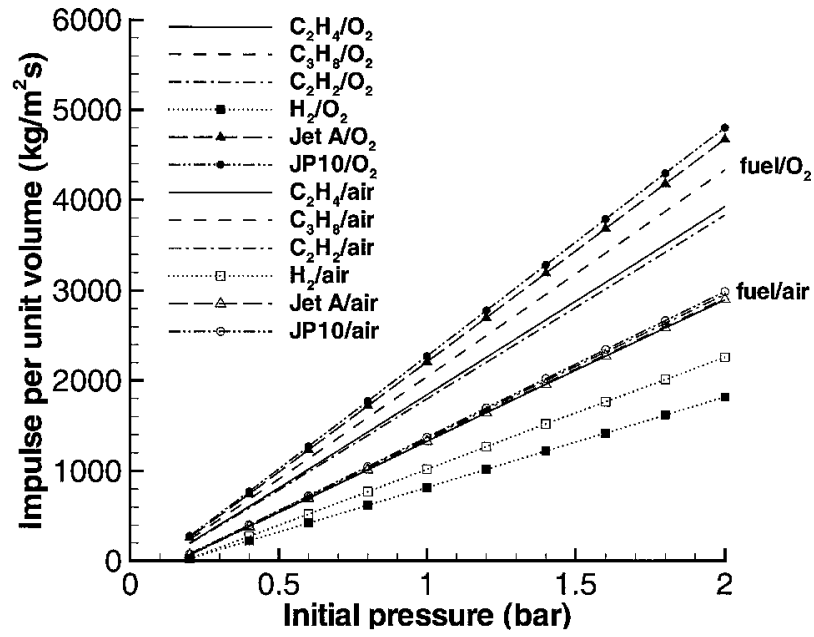

Fig. 17 Variation of impulse per unit volume with initial pressure; nominal initial conditions are $T_{1}=300 \mathrm{~K}$, stoichiometric fuel-oxygen ratio.

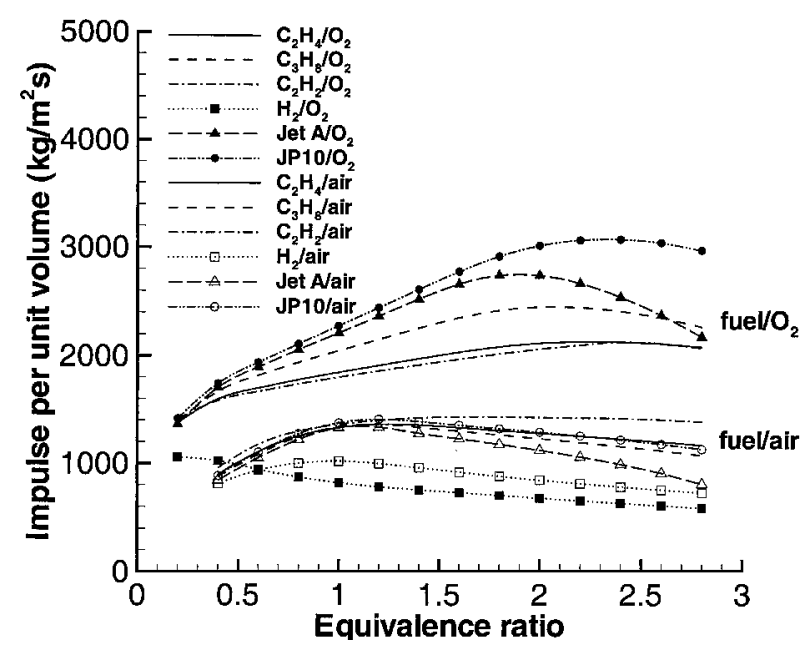

Fig. 18 Variation of impulse per unit volume with equivalence ratio; nominal initial conditions are $P_{1}=1$ bar and $T_{1}=300 \mathrm{~K}$.

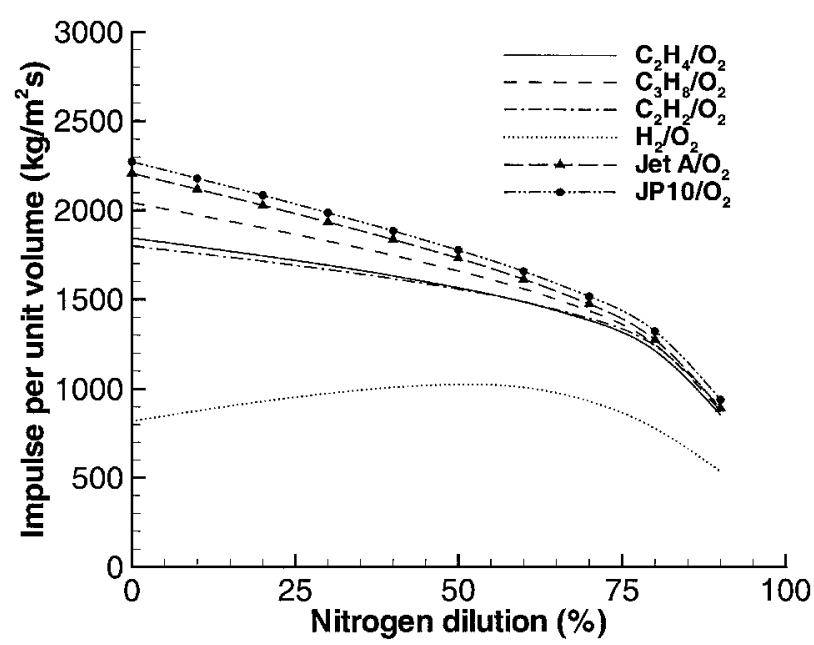

Fig. 19 Variation of impulse per unit volume with nitrogen dilution; nominal initial conditions are $P_{1}=1 \mathrm{bar}, T_{1}=300 \mathrm{~K}$, and stoichiometric fuel-oxygen ratio. 
the density of hydrogen mixtures is much lower, resulting in a lower impulse per unit volume.

Impulse per unit volume vs equivalenceratio is shown in Fig. 18. The impulse is expected to be maximum at stoichiometric conditions from Eq. (40) if we consider only the major products of combustion. However, examining Fig. 18, we see that, with the exception of hydrogen, the maximum values of $I_{V}$ occur for rich $(\phi \sim 2)$ fuel-oxygen mixtures and slightly rich $(\phi \sim 1.1-1.2)$ fuelair mixtures. Equilibrium computations reveal that the maximum detonation velocity and pressure also occur for rich mixtures. Even though the nominal heat of reaction of the mixture based on major products is maximum at stoichiometry, the detonation velocity is not at a maximum at stoichiometric conditions because of the product species distribution for rich combustion. Increasing amounts of $\mathrm{CO}$ and $\mathrm{H}_{2}$ in increasingly rich mixtures results in a larger number of products, effectively increasing the heat of reaction and shifting the peak detonation velocity and pressure to a rich mixture. The effect is much stronger in fuel-oxygen mixtures than in fuel-air mixtures because the nitrogen in the air moderates the effect of the increasing number of products in rich mixtures. A similar effect is observed in flames.

In the case of hydrogen, the product distribution effect is not as prominent because the number of major products is always less than reactants, independent of stoichiometry.For hydrogen-air mixtures, the maximum $I_{V}$ is obtained for an equivalence ratio close to 1. The impulse of hydrogen-oxygen mixtures decreases monotonically with increasing equivalence ratio. Unlike hydrocarbon fuels, which have a molecular mass comparable to or higher than oxygen and air, hydrogen has a much lower molecular mass. Thus, increasing the equivalence ratio causes a sharp decrease in the mixture density. The linear dependence of the impulse per unit volume with mixture density dominates over its square root variation with effective energy release [Eq. (40)], resulting in a decreasing impulse with increasing equivalence ratio for hydrogen-oxygen mixtures.

The impulse per unit volume generated by the different fuels with oxygen can be ranked in all cases, as follows, from lowest to highest: hydrogen, acetylene, ethylene, propane, Jet A, and JP10. The impulse is generated by the chemical energy of the mixture, which depends on a combination of bond strength and hydrogen to carbon ratio. The results obtained for the impulse per unit volume vs the equivalence ratio are presented for an equivalenceratio range from 0.4 to 2.6. The results of calculations at higher equivalence ratios were considered unreliable because carbon production, which is not possible to account for correctly in equilibrium calculations, occurs for very rich mixtures, in particular for Jet A and JP10.

The nitrogen dilution calculations (Fig. 19) show that the impulse decreases with increasing nitrogen dilution for hydrocarbon fuels. However, as the dilution increases, the values of the impulse for the different fuels approach each other. The presence of the diluent masks the effect of the hydrogen to carbon ratio. The hydrogen curve is much lower due to the lower CJ pressures caused by the lower molecular mass and heat of combustion of hydrogen. Unlike that for hydrocarbons, this curve has a maximum. The presence of this maximum can be explained by the two competing effects of nitrogen addition: One is to dilute the mixture, reducing the energy release per unit mass (dominant at high dilution), and the other is to increase the molecular mass of the mixture (dominant at low dilution). Note that the highest value of the impulse is obtained close to $50 \%$ dilution, which is similar to the case of air (55.6\% dilution).

\section{Mixture-Based Specific Impulse}

The mixture-based specific impulse $I_{\mathrm{sp}}$ is plotted vs initial pressure, equivalence ratio, and nitrogen dilution in Figs. 20, 21, and 22 , respectively. The specific impulse decreases steeply as the initial pressure decreases due to the increasing importance of dissociation at low pressures (Fig. 20). Dissociation is an endothermic process, and the effective energy release $q$ decreases with decreasing initial pressure.

Recombination of radical species occurs with increasing initial pressure. At sufficiently high initial pressures, the major products dominate over the radical species, and the $\mathrm{CJ}$ detonation properties

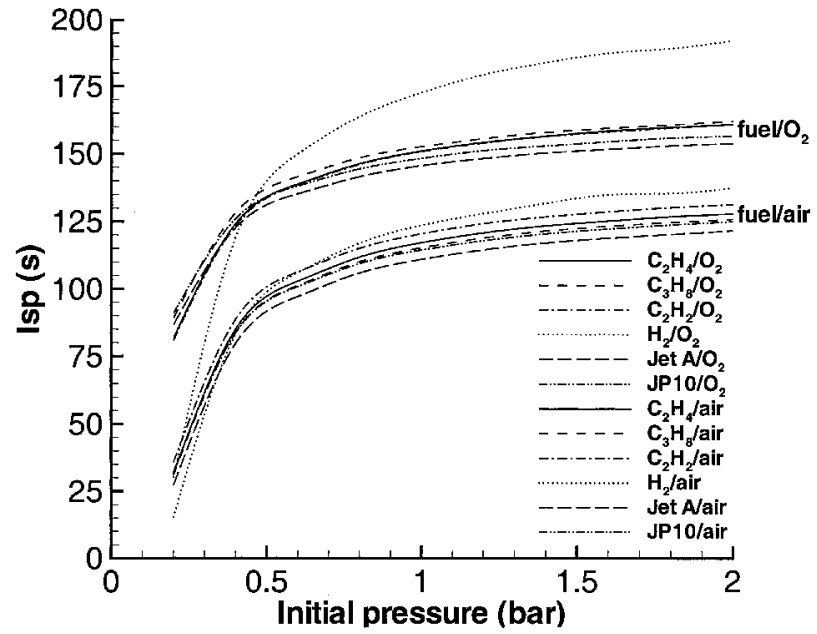

Fig. 20 Variation of mixture-based specific impulse with initial pressure; nominal initial conditions are $T_{1}=300 \mathrm{~K}$ and stoichiometric fueloxygen ratio.

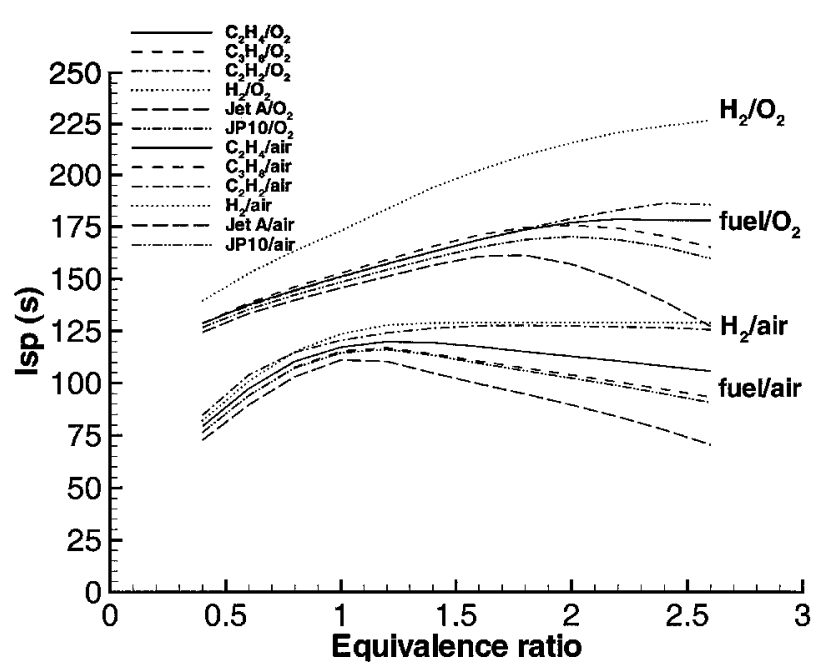

Fig. 21 Variation of mixture-based specific impulse with equivalence ratio; nominal initial conditions are $P_{1}=1$ bar and $T_{1}=300 \mathrm{~K}$.

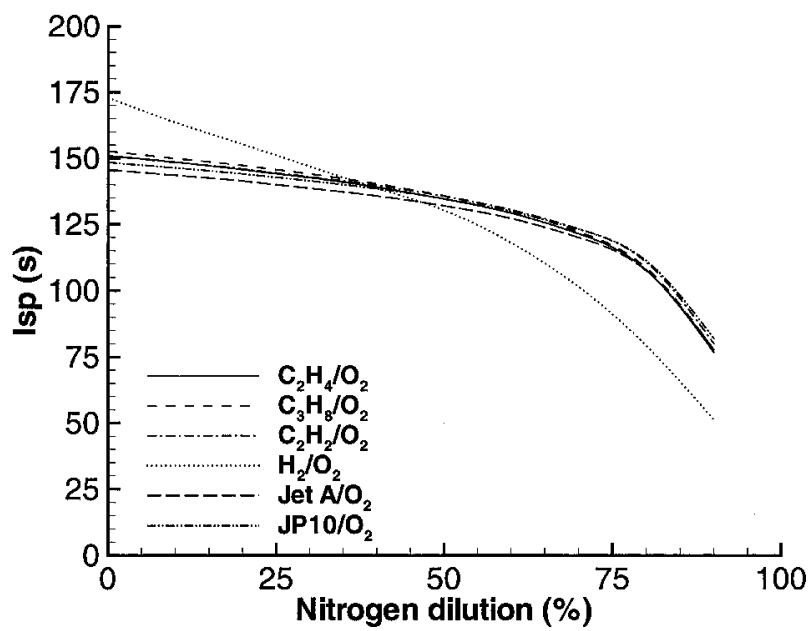

Fig. 22 Variation of mixture-based specific impulse with nitrogen dilution; nominal initial conditions are $P_{1}=1 \mathrm{bar}, T_{1}=300 \mathrm{~K}$, and stoichiometric fuel-oxygen ratio. 


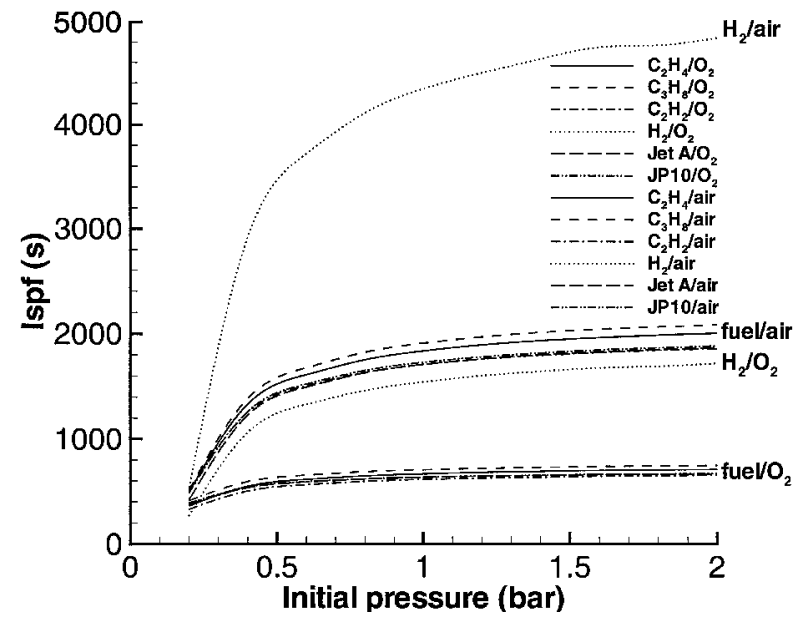

Fig. 23 Variation of fuel-based specific impulse with initial pressure; nominal initial conditions are $T_{1}=300 \mathrm{~K}$ and stoichiometric fuel-oxygen ratio.

tend to constant values. The mixture-based specific impulse tends to a constant value at high pressures, which is in agreement with the impulse scaling relationship of Eq. (43) if the values of $q$ and $\gamma$ reach limiting values with increasing initial pressure. Additional calculations for ethylene and propane with oxygen and air showed that the specific impulse was increased by approximately $7 \%$ between 2 and 10 bar and by less than $2 \%$ between 10 and 20 bar, confirming the idea of a high-pressure limit.

The specific impulses of hydrocarbon fuels varying the equivalence ratio (Fig. 21) have a similar behavior to that of the impulse per unit volume. This is expected because the only difference is due to the mixture density. Most hydrocarbon fuels have a heavier molecular mass than the oxidizer, but the fuel mass fraction for heavier fuels is smaller. The overall fuel mass in the mixture does not change much with the equivalence ratio, and so the mixture density does not vary significantly. However, this effect is important in the case of hydrogen, where the mixture density decreases significantly as the equivalenceratio increases. This accounts for the monotonic increase of the hydrogen-oxygen curve. In the case of hydrogen-air, the mixture density effect is masked because of the nitrogen dilution, which explains the nearly constant portion of the curve on the rich side. The variation of the $I_{\text {sp }}$ with nitrogen dilution (Fig. 22) is the same for all fuels, including hydrogen. The mixture-based specific impulse decreases as the nitrogen amount in the mixture increases.

\section{Fuel-Based Specific Impulse}

The fuel-based specific impulse $I_{\text {spf }}$ is plotted vs initial pressure, equivalence ratio, and nitrogen dilution in Figs. 23, 24, and 25, respectively. The variation of $I_{\text {spf }}$ with initial pressure (Fig. 23) is very similar to the corresponding behavior of $I_{\mathrm{sp}}$. The curves are individually shifted by a factor equal to the fuel mass fraction. Note the obvious shift of the hydrogen curves because of the very low mass fraction of hydrogen. The fuel-based specific impulse is about three times higher for hydrogen than for other fuels.

The plots in Fig. 24 show a monotonically decreasing $I_{\text {spf }}$ with increasing equivalence ratio. This is due to the predominant influence of the fuel mass fraction, which goes from low on the lean side to high on the rich side. The hydrogen mixtures again have much higher values compared to the hydrocarbon fuels due to the lower molar mass of hydrogen as compared to the hydrocarbonfuels. The values of $I_{\text {spf }}$ shown in Fig. 25 exhibit a monotonically increasing behavior with increasing nitrogen dilution, due to the decrease in fuel mass fraction as the nitrogen amount increases.

\section{Influence of Initial Temperature}

Temperature is an initial parameter that may significantly affect the impulse, especially at values representative of stagnation temperature for supersonic flight or temperatures required to vaporize

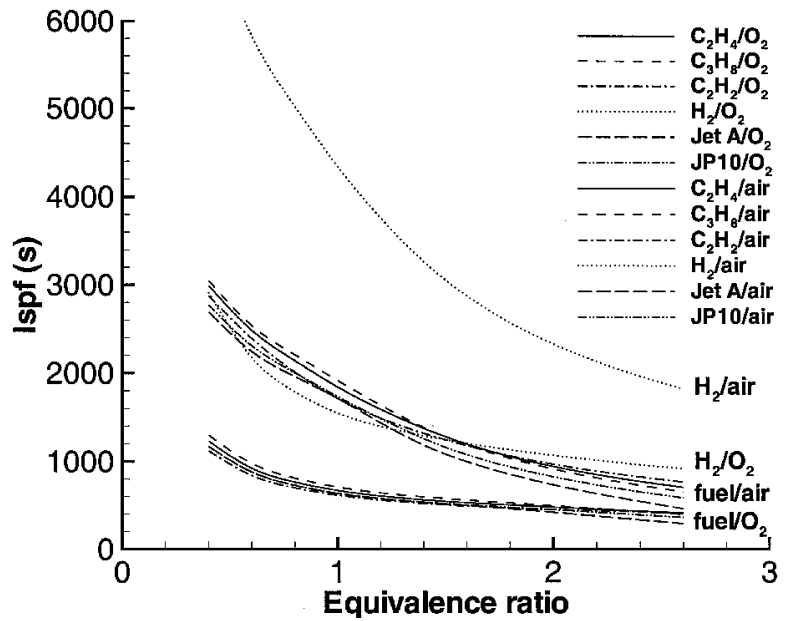

Fig. 24 Variation of fuel-based specific impulse with equivalence ratio; nominal initial conditions are $P_{1}=1$ bar and $T_{1}=300 \mathrm{~K}$.

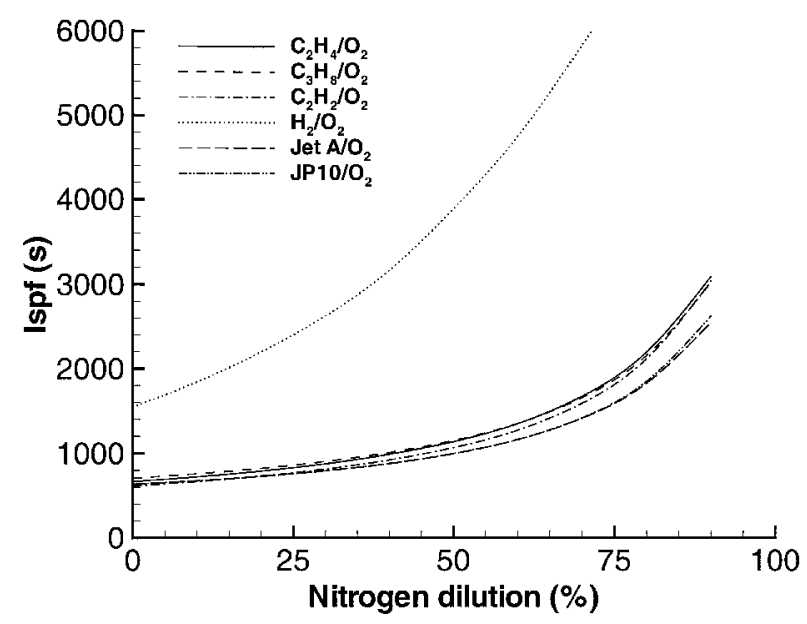

Fig. 25 Variation of fuel-based specific impulse with nitrogen dilution; nominal initial conditions are $P_{1}=1 \mathrm{bar}, T_{1}=300 \mathrm{~K}$, and stoichiometric fuel-oxygen ratio.

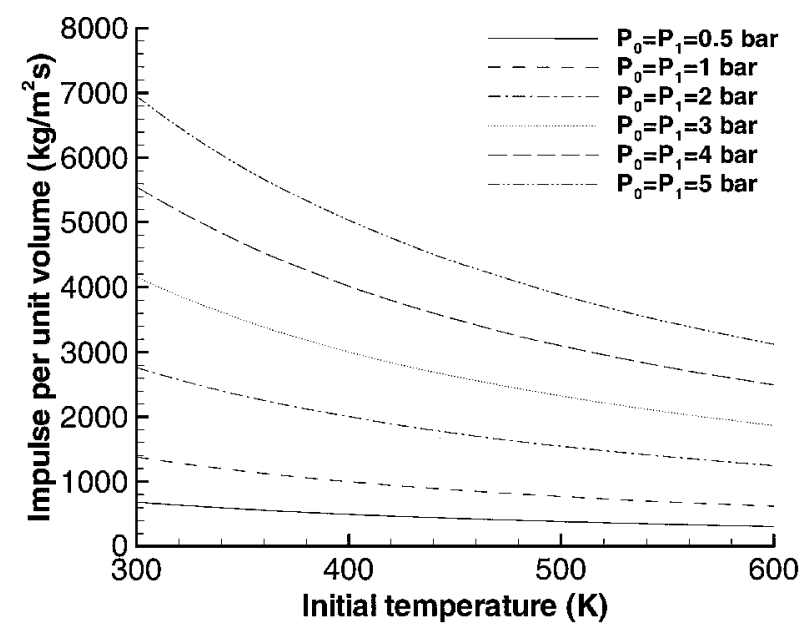

Fig. 26 Variation of impulse per unit volume with initial temperature for different values of the stagnation pressure.

aviation fuels. The results shown in Figs. 17-25 were for an initial temperature of $300 \mathrm{~K}$. Calculations with initial temperatures from 300 to $600 \mathrm{~K}$ were carried out for stoichiometric JP10-air; JP10 is a low vapor pressure liquid $\left(\mathrm{C}_{10} \mathrm{H}_{16}\right)$ at room temperature. The impulse per unit volume (Fig. 26) and the mixture-based specific impulse (Fig. 27) were calculated as a function of the initial temperature for different pressures representative of actual stagnation pressure values in a real engine. 


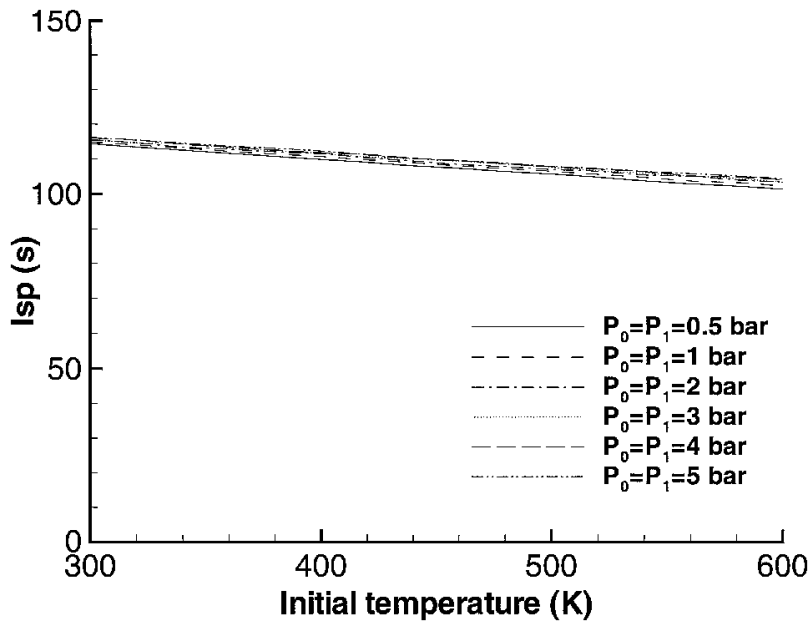

Fig. 27 Variation of mixture-based specific impulse with initial temperature for different values of the stagnation pressure.

The impulse per unit volume decreases with increasing initial temperature, as predicted by Eq. (42). At fixed pressure and composition, this decrease is caused by the decrease of the initial mixture density. The mixture-based specific impulse is found to be approximately constant when initial temperature and initial pressure are varied (Fig. 27). The scaling predictions of Eq. (43) are verified for constant composition. The slight decrease of the specific impulse observed with increasing temperature and decreasing pressure can be attributed to the promotion of dissociation reactions under these conditions. Specific impulse is a useful parameter for estimating performance because, at high enough initial pressures, it is almost independent of initial pressure and temperature.

\section{Conclusions}

An analytical model for the impulse of a pulse detonation tube has been developed using a simple one-dimensional gasdynamic analysis and empirical observations. The model offers the possibility to evaluate, in a simple way, the performance of the most basic form of a PDE consisting of a straight tube open at one end. The model predictions were compared with various experimental results, from direct single-cycle impulse measurements ${ }^{8,9}$ to multicycle thrust measurements, ${ }^{23,24}$ as well as with numerical simulations. These show reasonable agreement (within $\pm 15 \%$ or better in most cases) for comparisons of impulse per unit volume, specific impulse, and thrust. Parametric calculations were conductedfor a wide range of initial conditions, including fuel type (hydrogen, acetylene, ethylene, propane, Jet A, and JP10), initial pressure (from 0.2 to 2 bar), equivalence ratio (from 0.4 to 2.6), and nitrogen dilution (from 0 to $90 \%$ ).

The impulse of a detonation tube was found to scale directly with the mass of the explosive mixture in the tube and the square root of the effective energy release per unit mass of the mixture. A procedure was given to account for product dissociation in determining the effective specific energy release. We derived scaling relationships and carried out equilibrium computations to verify the following conclusions:

1) At fixed composition and initial temperature, the impulse per unit volume varies linearly with initial pressure.

2) At fixed composition and initial pressure, the impulse per unit volume varies inversely with initial temperature.

3) At fixed composition and sufficiently high initial pressure, the specific impulse is approximately independent of initial pressure and initial temperature. This makes specific impulse the most useful parameter for estimating pulse detonation tube performance over a wide range of initial conditions.

The predicted values of the mixture-based specific impulse are on the order of $150 \mathrm{~s}$ for hydrocarbon-oxygen mixtures, $170 \mathrm{~s}$ for hydrogen-oxygen, and on the order of 115-130 s for fuel-air mixtures at initial conditions of $1 \mathrm{bar}$ and $300 \mathrm{~K}$. These values are lower than the maximumimpulses possible with conventionalsteady propulsiondevices. As mentioned in the introduction, there are many other factors that should be considered in evaluating PDE performance and their potential applications. The present study provides some modeling ideas that could be used as a basis for more realistic engine simulations.

\section{Acknowledgments}

This work was supported by the Office of Naval Research Multidisciplinary University Research Initiative Multidisciplinary Study of Pulse Detonation Engine (Grant 00014-99-1-0744, Subcontract 1686-ONR-0744), and General Electric contract GE-PO A02 81655 under DABT-63-0-0001. We are grateful to Hans Hornung for the numerical simulations. We thank F. Schauer at the U.S. Air Force Research Laboratory for sharing his data with us.

\section{References}

${ }^{1}$ Sterling, J., Ghorbanian, K., Humphrey, J., Sobota, T., and Pratt, D., "Numerical Investigations of Pulse Detonation Wave Engines," AIAA Paper 95-2479, July 1995 .

${ }^{2}$ Bussing, T. R. A., and Pappas, G., "Pulse Detonation Engine Theory and Concepts," Developments in High-Speed Vehicle Propulsion Systems, Vol. 165, Progress in Aeronautics and Astronautics, AIAA, Reston, VA, 1996, pp. 421-472.

${ }^{3}$ Bussing, T. R. A., Bratkovich, T. E., and Hinkey, J. B., "Practical Implementation of Pulse Detonation Engines," AIAA Paper 97-2748, July 1997.

${ }^{4}$ Cambier, J. L., and Tegner, J. K., "Strategies for Pulsed Detonation Engine Performance Optimization," Journal of Propulsion and Power, Vol. 14, No. 4, 1998, pp. 489-498.

${ }^{5}$ Kailasanath, K., and Patnaik, G., "Performance Estimates of Pulsed Detonation Engines," Proceedings of the 28th International Symposium on Combustion, Combustion Inst., Pittsburgh, PA, 2000, pp. 595-601.

${ }^{6}$ Zhdan, S. A., Mitrofanov, V. V., and Sychev, A. I., "Reactive Impulse from the Explosion of a Gas Mixture in a Semi-infinite Space," Combustion, Explosion and Shock Waves, Vol. 30, No. 5, 1994, pp. 657-663.

${ }^{7}$ Heiser, W. H., and Pratt, D. T., "Thermodynamic Cycle Analysis of Pulse Detonation Engines," Journal of Propulsion and Power, Vol. 18, No. 1, 2002, pp. 68-76.

${ }^{8}$ Zitoun, R., and Desbordes, D., "Propulsive Performances of Pulsed Detonations," Combustion Science and Technology, Vol. 144, Nos. 1-6, 1999, pp. $93-114$

${ }^{9}$ Cooper, M., Jackson, S., Austin, J., Wintenberger, E., and Shepherd, J. E., "Direct Experimental Impulse Measurements for Deflagrations and Detonations," AIAA Paper 2001-3812, July 2001.

${ }^{10}$ Harris, P. G., Farinaccio, R., and Stowe, R. A., "The Effect of DDT Distance on Impulse in a Detonation Tube," AIAA Paper 2001-3467, July 2001.

${ }^{11}$ Nicholls, J. A., Wilkinson, H. R., and Morrison, R. B., "Intermittent Detonation as a Thrust-Producing Mechanism," Jet Propulsion, Vol. 27, No. 5, 1957, pp. 534-541

${ }^{12}$ Endo, T., and Fujiwara, T., "A Simplified Analysis on a Pulse Detonation Engine," Transactions of the Japanese Society for Aeronautical and Space Sciences, Vol. 44, No. 146, 2002, pp. 217-222.

${ }^{13}$ Stanyukovich, K. P., Unsteady Motion of ContinuousMedia, Pergamon, New York, 1960, pp. 142-196.

${ }^{14}$ Kailasanath, K., Patnaik, G., and Li, C., "Computational Studies of Pulse Detonation Engines: A Status Report,” AIAA Paper 1999-2634, June 1999.

${ }^{15}$ Kailasanath, K., "Recent Developments in the Research on Pulse Detonation Engines," AIAA Paper 2002-0470, Jan. 2002.

${ }^{16}$ Zel'dovich, Y. B., "On the Theory of the Propagation of Detonations in Gaseous Systems," Journal of Experimental and Theoretical Physics, Vol. 10, 1940, pp. 542-568; translation as NACA TM 1261, 1950

${ }^{17}$ Taylor, G. I., "The Dynamics of the Combustion Products behind Plane and Spherical Detonation Fronts in Explosives," Proceedings of the Royal Society of London, Series A: Mathematical and Physical Sciences, Vol. A200, 1950, pp. 235-247.

${ }^{18}$ Reynolds, W. C., "The Element Potential Method for Chemical Equilibrium Analysis: Implementation in the Interactive Program STANJAN, Version 3," Technical Rept., Dept. of Mechanical Engineering, Stanford Univ., Stanford, CA, Jan. 1986.

${ }^{19}$ Thompson, P. A., Compressible Fluid Dynamics, Rensselaer Polytechnic Inst. Bookstore, Troy, NY, 1988, pp. 347-359.

${ }^{20}$ Fickett, W., and Davis, W. C., Detonation Theory and Experiment, Dover, New York, 2001, Chap. 2, pp. 16-20.

${ }^{21}$ Glass, I. I., and Sislian, J. P., Nonstationary Flows and Shock Waves, Oxford Science Publ., Clarendon Press, Oxford, England, U.K., 1994, Chap. 4.

${ }^{22}$ Quirk, J. J., "AMRITA-A Computational Facility (for CFD Modelling)," VKI 29th CFD Lecture Series, edited by H. Deconind, von Kármán Inst. for Fluid Dynamics, Belgium, 1998. 
${ }^{23}$ Schauer, F., Stutrud, J., and Bradley, R., "Detonation Initiation Studies and Performance Results for Pulsed Detonation Engines," AIAA Paper 20011129, Jan. 2001

${ }^{24}$ Schauer, F., Stutrud, J., Bradley, R., Katta, V., and Hoke, J., "Detonation Initiation and Performance in Complex Hydrocarbon Fueled Pulsed Detonation Engines," JANNAF, Paper I-05, July 2001.

${ }^{25}$ Shepherd, J. E., and Kaneshige, M., "Detonation Database," Graduate Aeronautical Labs., GALCIT Rept. FM97-8, California Inst. of Technology, Pasadena, CA, 1997; rev. 2001, see URL: http://www.galcit.caltech.edu/ detn_db/html.

${ }^{26}$ Zel'dovich, Y., Kogarko, S., and Simonov, N., "An Experimental Investigation of Spherical Detonation," Soviet Physics-Technical Physics, Vol. 1, No. 8, 1956, pp. 1689-1713.
${ }^{27}$ Lee, J., "Dynamic Parameters of Gaseous Detonations," Annual Review of Fluid Mechanics, Vol. 16, 1984, pp. 311-316.

${ }^{28}$ Dorofeev, S., Kuznetsov, M., Alekseev, V., Efimenko, A., and Breitung, W., "Evaluation of Limits for Effective Flame Acceleration in Hydrogen Mixtures," Journal of Loss Prevention in the Process Industries, Vol. 14, No. 6, 2001, pp. 583-589.

${ }^{29}$ Dorofeev, S., Sidorov, V. P., Kuznetzov, M. S., Matsukov, I. D., and Alekseev, V. I., "Effect of Scale on the Onset of Detonations," Shock Waves, Vol. 10, No. 2, 2000, pp. 137-149.

${ }^{30}$ Chao, T., Wintenberger, E., and Shepherd, J. E., "On the Design of Pulse Detonation Engines," Graduate Aeronautical Labs., GALCIT Rept. FM00-7, California Inst. of Technology, Pasadena, CA, 2001. 